\title{
LAS CONTRIBUCIONES DE LA ACADEMIA CHINA Y JAPONESA EN LA TEORÍA DE LAS RELACIONES INTERNACIONALES. MAS ALLÁ DEL DOMINIO OCCIDENTAL
}

\author{
Lluc López i Vidal ${ }^{1}$ Iván González-Pujol $^{2}$ Ferran Pérez-Mena $^{3}$ \\ Universitat Oberta de Catalunya/ Universidad de Senshu/ Universitat Oberta de Catalunya \\ Universidad de Sussex
}

\begin{abstract}
Resumen:
El desarrollo teórico de las Relaciones Internacionales se encuentra dominado por la academia occidental; no obstante, las teorías autóctonas han tomado un papel destacado en la oposición a dicha dominación. Con el objetivo de realizar una revisión crítica de la literatura sobre las teorías no occidentales se han identificado y analizado como casos de estudio las teorías autóctonas producidas en China y en Japón. En el caso de la academia China concluimos que su desarrollo teórico cuestiona el predominio occidental-céntrico y ha empezado a combatir la hegemonía euroamericana. En cambio, la evolución teórica de la disciplina en Japón avanza en los momentos en que el país se posiciona como potencia en ascenso, mientras que se estanca en períodos en que el país se acomoda al statu quo.
\end{abstract}

Palabras Clave: Relaciones Internacionales, estudios asiáticos, historia japonesa, historia china, teoría política

Title in English: The Contributions of the Chinese and Japanese Academy in the Theory of International Relations: Beyond Western Domination

\section{Abstract:}

Theoretical development of the International Relations discipline has been dominated by the Western production of knowledge; however, indigenous theories have been taking a prominent opposition stance to that domination. With the aim of providing a critical review about the literature on NonWestern IR theory, we have identified and analysed the case study of indigenous theories in China and Japan. On the one hand, we conclude that the theoretical innovations produced by the Chinese IR academia challenge the western dominance and it has started to counterbalance Western hegemony. On the other hand, the theoretical evolution in Japan shows a trend of advancement when Japan plays a rising power role, but stalls in periods of accommodation with the statu quo.

Key Words: International relations, Asian studies, Japanese history, Chinese history, political theory

Copyright $\odot$ UNISCI, 2019.

Las opiniones expresadas en estos artículos son propias de sus autores, y no reflejan necesariamente la opinión de UNISCI. The views expressed in these articles are those of the authors, and do not necessarily reflect the views of UNISCI.

\footnotetext{
${ }^{1}$ Lluc López i Vidal es Profesor titular de Relaciones Internacionales y director del Máster en Cooperación Internacional al Desarrollo y Acción Humanitaria en la Universitat Oberta de Catalunya.

E-mail:<llopezvi@uoc.edu>

${ }^{2}$ Iván González-Pujol es investigador predoctoral en la Universidad de Senshu. Máster en Estudios de Asia Oriental por la Universidad de Salamanca y el Máster en Dirección de las Organizaciones en la Economía del Conocimiento por la Universitat Oberta de Catalunya.

E-mail: <igp@uoc.edu>

${ }^{3}$ Ferrán Pérez-Mena es Profesor asociado del Master International Affairs and Diplomacy en la Universitat Oberta de Catalunya. Máster en Estudios de Asia Pacífico por la Universidad Nacional de Chengchi e investigador doctoral en la Universidad de Sussex.

E-mail:<fp200@sussex.ac.uk>

DOI: http://dx.doi.org/10.31439/UNISCI-67
} 


\section{Introducción}

En los últimos años han aparecido un gran número de publicaciones que han evidenciado la naturaleza occidentalcéntrica ${ }^{4}$ que caracteriza la disciplina de las Relaciones Internacionales (RRII) desde su fundación a principios del siglo pasado. ${ }^{5}$ El cuerpo teórico de la disciplina se ha ido cimentando sobre la base de unas tradiciones de pensamiento ${ }^{6} \mathrm{y}$ de unos debates paradigmáticos que adolecen de una narrativa netamente occidental. Los orígenes y la evolución de la disciplina atestiguan un etnocentrismo que ha producido unos efectos homogeneizadores a escala global. Buena parte de los constructos teóricos y modelos analíticos se han planteado desde un pretendido universalismo de las Ciencias Sociales, a pesar de estar limitados a la experiencia de Europa Occidental y Norteamérica. En una academia construida desde y para Occidente, ${ }^{7}$ esta perspectiva occidentalocéntrica ha producido una situación de clara desventaja para las academias de algunos países situados fuera de esta zona. Todo ello ha

\footnotetext{
${ }^{4}$ En este artículo utilizaremos el vocablo occidenalcéntrico como término neutro para hablar de la hegemonía académica tanto de Europa Occidental como de Norteamérica. Aunque existen otros términos como americanocéntrico, norteamericanocéntrico, eurocéntrico o euroamericanocéntrico, el uso de occidentalocéntrico evita entrar en el debate sobre si la disciplina es más norteamericanocéntrica o eurocréntrica. En los casos en que en el artículo se utilicen los términos euroamericanismo o americanismo, se pretende enfatizar el peso de una u otra región. Para un análisis cuantitativo sobre el uso de dichos términos vid. Wemheuer-Vogelaar, Wiebke; Bell, Nicholas J.; Navarrete Morales Mariana y Tierney, Michael J.: "The IR of the beholder: examining global IR using the 2014 TRIP Survey”, International Studies Review, vol. 18, nº 1 (marzo 2016), pp. 16-32.

${ }^{5}$ Aunque existe una literatura muy extensa sobre la cuestión, algunos de los autores que lo han abordado desde una perspectiva crítica han sido Acharya, Amitav y Buzan, Barry (eds.) (2010): Non-Western International Relations Theory: perspectives on and beyond Asia, London, Routledge; Cox, Robert W.: "Social Forces, States and World Orders: Beyond International Relations Theory", Millennium, vol. 10, n 2 (junio 1981), pp. 126-155; Doty, Roxanne Lynn (1996): Imperial Encounters: the Politics of Representation in North-South Relations, Minneapolis, University of Minnesota Press; Hobson, John M. (2012): The Eurocentric Conception of World Politics: Western International Theory, 1760-2010, New York, Cambridge University Press; Gruffydd Jones, Branwen (ed.) (2006): Decolonizing International Relations, Lanham, Rowman \& Littlefield; Krishna, Sankaran: "The Importance of Being Ironic: A Postcolonial View on Critical International Relations Theory", Alternatives: Global, Local, Political, vol. 18, nº 3 (julio 1993), pp. 385-417; Ling, L. H. M. (2002): Postcolonial International Relations: Conquest and Desire between Asia and the West, New York, Palgrave; Qin, Yaqing: "Why is there no Chinese International Relations Theory?" International Relations of the Asia-Pacific, vol. 7, $n^{\circ} 3$ (septiembre 2007), pp. 313-340; Tickner, Arlene B. y Wæver, Ole (eds.) (2009): International Relations Scholarship around the World, New York, Routledge; o Tickner, Arlene B. y Blaney, David. L. (eds.) (2012): Thinking International Relations Differently, New York, Routledge. En lengua castellana, en los últimos años han aparecido un número cada vez más importante de investigaciones sobre el etnocentrismo en las RRII. Algunos ejemplos recientes los encontramos en Del Arenal, Celestino. (2014): Etnocentrismo y Teoría de las Relaciones Internacionales: una visión crítica, Madrid, Editorial Tecnos; Galindo Rodríguez, Fernando: "Enfoques postcoloniales en Relaciones Internacionales: un breve recorrido por sus debates y sus desarrollos teóricos", Relaciones Internacionales, vol. 22 (febrero 2013), pp. 85-107; Lorenzini, María Elena y Pereyra Doval, María Gisela: "Revisitando los aportes de las teorías del sur: nexos entre teoría y praxis en Argentina y Brasil", Relaciones Internacionales, vol. 22 (febrero 2013), pp. 9-26; Montobbio, Manuel: “Tianxia, Teoría de las Relaciones Internacionales y ascenso global de China”, Revista Española de Derecho Internacional, vol. 70, nº 1 (enero-junio 2018), pp. 235-246; Moure, Leire (2014): “Orden internacional en transición y Relaciones Internacionales: Aproximaciones teóricas al declive hegemónico estadounidense y al ascenso de China como potencia global", en Cursos de Derecho Internacional y Relaciones Internacionales de Vitoria-Gasteiz 2013, Madrid, Thompson Reuters-Aranzadi, o Pintado Lobato, Montse: "Hacia una teoría china de las relaciones internacionales: Evolución, proyectos teóricos y pertinencia práctica”, Revista Española de de Derecho Internacional, vol. 70, nº 1 (enero-junio 2018), pp. 201-226.

${ }^{6}$ Martin Wight, uno de los miembros más destacados de la Escuela Inglesa de las RRII, diferenciaba tres grupos de pensadores o tradiciones: los maquiavélicos o realistas, los grocianos o racionalistas y los kantianos o revolucionarios. Sobre estas tradiciones vid. Wight, Martin, Wight, Gabriele (ed.) y Porter, Brian (ed.) (1992): International theory: the Three Traditions, New York, Holmes \& Meier.

${ }^{7}$ Entre los autores más citados en los manuales de RRII se suelen mencionar a Tucídides, Maquiavelo, Hobbes, Kant, Grocio, Bentham, Locke o Marx por nombrar solo algunos de los más importantes. Sobre esta cuestión vid. Del Arenal, Celestino y Sanahuja, José Antonio (eds.) (2015): Teorías de las Relaciones Internacionales, Madrid, Tecnos.
} 
provocado que la academia no occidental haya empezado a denunciar dicha situación de dominio y haya reivindicado la necesidad de "descolonizar, descentralizar y pluralizar" una disciplina que requiere de nuevas herramientas conceptuales y analíticas para aprehender los grandes problemas de la realidad internacional. ${ }^{8}$

Las causas que apuntan al dominio de Occidente en la disciplina han sido abordadas en los estudios ya clásicos de Amitav Acharya y Barry Buzan, Chan y Mandaville,Tickner y Blaney, y Tickner y Wæver. ${ }^{9}$ En las obras de dichos autores converge una narrativa común alrededor de la idea de que el occidentalcentrismo busca el predominio tanto de la filosofía occidental como de la visión eminentemente europea de la Historia, una afirmación que asume que Occidente ha encontrado "el camino adecuado" para entender las RRII. ${ }^{10}$ Asimismo, dicho dominio es el resultado del estatus hegemónico que las academias euroamericanas de las RRII han adquirido durante todo el siglo XX. Reformulado en términos gramscianos, la adquisición de dicho estatus ha producido una posición de predominio intelectual que ha terminado reforzando la narrativa sobre la pretendida inclinación universalista de las RRII. ${ }^{11}$ En otros casos, dicho predominio no responde tanto a la inexistencia de teorías no occidentales, ${ }^{12}$ sino a la marginalización de las teorías no occidentales tanto en la comunidad internacional como en sus propios contextos locales. En definitiva, las causas de dicho predominio, ya sea por su posición hegemónica, como por la invisibilidad de las narrativas fuera de Occidente, son el resultado del inmenso poder militar, económico y, por ende, cultural acumulado por Europa en los últimos tres siglos de la Historia. Como afirma Del Arenal y Sanahuja, ${ }^{13}$ este poder ha supuesto el desarrollo de unas Ciencias Sociales marcadamente occidentales y sin apenas competencia.

La nueva distribución de poder en un sistema internacional que está en completa mutación obliga a plantearnos cómo se está reconfigurando el campo de las ideas y, en

\footnotetext{
8 Tickner, Arlene B.: "Core, periphery and (neo)imperialist International Relations", European Journal of International Relations, vol. 19, $\mathrm{n}^{\mathrm{o}} 3$ (septiembre 2013), pp. 627-646.

${ }^{9}$ Ibid.; Acharya et al.: Non-Western international relations theory, op. cit.; Chan, Stephen y Mandaville, Peter G. (eds.) (2001): The Zen of International Relations. IR Theory from East to West, London, Palgrave Macmillan; Tickner et al. Thinking International Relations Differently, op. cit. y Tickner et al. International Relations Scholarship around, op. cit.

${ }^{10}$ Acharya et al.: Non-Western international relations theory, op. cit. Sobre el origen eurocéntrico de la disciplina y la descolonización del conocimiento vid. Gruffydd Jones, op. cit. y Agathangelou, Anna M. y Ling, L. H. M.: "The House of IR: From Family Power Politics to the Poisies of Worldism", International Studies Review, vol. 6, $\mathrm{n}^{\circ} 4$ (diciembre 2004), pp. 21-50.

${ }^{11}$ Acharya et al. Non-Western international relations theory, op. cit.

${ }^{12}$ Por razones de simplificación conceptual utilizamos el término "no occidental", aunque en la disciplina se ha debatido en profundidad sobre el uso de dicho vocablo. "No occidental" se ha utilizado para identificar a toda aquella teoría producida por autores que no conforman el mainstream situado en los Estados Unidos y, en menor medida, en Europa Occidental. Sin embargo, el uso del vocablo "no occidental" para definir grandes conceptos geográficos como Asia o América resulta demasiado impreciso, tal y como el presente trabajo trata de demostrar. Tickner y Blaney (2012) utilizan una nomenclatura que resulta mucho más apropiada: "non-core scholars", es decir, aquellos autores que residen y trabajan en instituciones que no pertenecen al centro de producción científica de la disciplina. La distinción entre centros académicos "core" —aquellos autores que trabajan en países occidentales - y "non-core" permite incorporar a autores que, a pesar de trabajar en países no occidentales, comparten y participan de la epistemología occidental. Por otra parte, en la revisión del clásico trabajo de Acharya y Buzan (2007) realizada en julio de 2017 en la revista International Relations of Asia Pacific, Acharya y Buzan apuestan por un nuevo vocablo que supere los límites de la etiqueta "no occidental" y utilizan la expresión "Global IR", una expresión más integradora que evita dicha dicotomía. Vid. Acharya, Amitav y Buzan, Barry: "Why is there no Non-Western International Relations Theory? Ten years on", International Relations of the Asia-Pacific, vol. 17, $\mathrm{n}^{\mathrm{o}} 3$ (septiembre 2017), y Acharya, Amitav y Buzan, Barry: "Why is there no non-Western international relations theory? An introduction”, International Relations of the Asia-Pacific, vol. 7, $\mathrm{n}^{\circ} 3$ (septiembre 2007), pp. 287-312 y Tickner et al. Thinking International Relations Differently, op. cit.

${ }^{13}$ Del Arenal et al., op. cit.
} 
particular, cómo se amplían los debates de nuestra disciplina. El presente artículo analiza el papel de las academias china ${ }^{14}$ y japonesa en los debates de la disciplina de las RRII y trata de responder a la pregunta sobre si dichas academias desafían el discurso occidentalocéntrico dominante. En el caso chino, la teoría autóctona apunta hacia la necesidad de construir un marco analítico propio y diferenciado con el que cuestionar y combatir la hegemonía de la teoría occidental. En el caso japonés, en cambio, la aportación de dicha academia trasciende la simple verificación de teorías occidentales e intenta ampliar la teoría general de las RRII sin rivalizar con las teorías dominantes. Más que luchar contra el discurso occidentalcéntrico, la academia japonesa trata de añadir matices y colores en la paleta del teórico occidental.

El objetivo de este artículo es identificar las principales construcciones teóricas autóctonas tanto en China como en Japón, y analizar el rol que cada uno de estos países está adquiriendo en la disciplina. Ambos casos resultan relevantes puesto que, por una parte, dos países de una misma región con unos contextos históricos y culturales específicos han producido distintas y, a veces, antagónicas teorías sobre las RRII. Por la otra parte, estos dos casos resultan esenciales para entender las consecuencias que la expansión de la modernidad en Asia Oriental ha producido en dos de los países claves en la región. En el primer caso, estudiaremos como, desde la apertura económica de China en 1978 y coincidiendo con el "giro civilizatorio", ${ }^{15}$ el sector crítico de la academia china ha promovido nuevas ideas y conceptos para explicar las RRII. Para ello, dicha academia ha utilizado epistemologías derivadas del pensamiento político chino y ha rehusado la naturaleza occidentalcéntrica de la disciplina. En el estudio de caso identificaremos el nacimiento de la disciplina y trataremos de entender qué elementos constituyen la creación de teorías y escuelas en China y si estas se basan en modelos científicos occidentales positivistas o en modelos pospositivistas. Particularmente, analizaremos las tres principales escuelas que han surgido en la academia china en los últimos 20 años: la teoría del Tianxia (天下) de Zhao Tingyang, el Realismo Moral de Yan Xuetong y la "Teoría de la Relacionalidad constructivista" de Qin Yaqing. Como veremos a lo largo del artículo, estas tres teorías han contribuido tanto a la reafirmación el discurso del ascenso pacífico de China en el orden liberal, como a la adquisición de una serie de herramientas necesarias para afrontar y guiar los desafíos que surgen de la construcción de un orden internacional alternativo.

En el caso de Japón, a pesar de que el país se encuentra geográficamente en una región considerada no occidental, la disciplina de las RRII se origina principalmente con la importación de las tradiciones de pensamiento europeas a partir de la Restauración Meiji (1868), y se complementará en una segunda fase de introducción de conocimiento occidental tras la Segunda Guerra Mundial. Por sus fundamentos teóricos ligados a las tradiciones occidentales, podríamos presuponer que la formulación teórica japonesa no goza de elementos autóctonos propios. Sin embargo, como demuestra este artículo, la academia japonesa ha encontrado un punto intermedio entre la occidentalización y la no-occidentalización. La modernización de Japón se ha modulado siguiendo unos patrones eminentemente occidentales, pero manteniendo una alta presencia de la distinción japonesa, algo a lo que nos referiremos,

\footnotetext{
${ }^{14}$ Cuando el presente artículo menciona a China o a su academia hace referencia a la República Popular de China.

${ }^{15}$ El "civilizational turn of IR" es una corriente académica nacida en los años 2000 que trata de contrarrestar los argumentos del libro de Samuel Huntington El choque de civilizaciones. Esta corriente tuvo un gran impulso después de que el presidente de Irán, Mohammad Khatami, hablase sobre el "Diálogo de civilizaciones" en el año 2001. La literatura sobre el giro civilizatorio aborda como temas centrales los "encuentros civilizatorios", las "interconexiones entre civilizaciones" y cómo éstas influyen en las RRII. Para un mayor detalle vid. Michael, Michaelis S. y Petito, Fabio (eds.) (2009): Civilizational Dialogue and World Order: The Other Politics of Cultures, Religions, and Civilizational in International Relations, Nueva York, Palgrave Macmillan.
} 
en palabras de Ikeda Josuke, ${ }^{16}$ como in-between-ness. En este estudio nos centraremos en dos de las teorías japonesas más relevantes en la disciplina durante el siglo XX y que han fundamentado la política exterior japonesa del periodo imperialista: la teorización de la Esfera de Coprosperidad de la Gran Asia Oriental, cuyas principales contribuciones provinieron de la escuela de Kyoto ${ }^{17}$ y del filósofo Rōyama Masamichi, y el modelo de los "gansos voladores" del economista Akamatsu Kaname. Veremos cómo estas dos teorías intentan establecer una identidad japonesa en un punto intermedio entre Oriente y Occidente.

El artículo está divido en tres partes. En la primera parte se analizará el caso de la academia china, desde su nacimiento, hasta el pensamiento político del presidente Xi Jinping. En la segunda parte se estudiará el caso japonés, identificando las diferentes tradiciones vinculadas con las RRII durante el siglo XX, las diferentes teorías de alcance medio hasta los fundamentos teóricos de la política exterior del Primer Ministro Shinzo Abe. Tras el análisis de estos dos casos, en el último apartado apuntaremos algunas conclusiones finales.

\section{La disciplina de las RRII en China}

Desde la apertura económica del país en 1978, el estudio de la disciplina de las RRII ha experimentado un gran auge en China. Durante estos últimos treinta años, los académicos chinos se han dedicado a estudiar diversos temas que hasta la fecha no habían sido examinados en profundidad. Una de las cuestiones más debatidas entre los teóricos chinos ha sido la identificación de la fecha exacta en la que nació la disciplina en su país. ${ }^{18} \mathrm{La}$ comunidad académica china ha sugerido que la disciplina se originó en la década de los ochenta, coincidiendo con la apertura económica de China y el inicio del "socialismo con características chinas". Durante las décadas previas a la apertura del país, las Ciencias Sociales estuvieron dominadas por la narrativa propia del marxismo-leninismo y el maoísmo y el estudio de las RRII se limitaba a interpretar a los clásicos revolucionarios - Marx, Engels, Lenin, Stalin o Mao Zedong - con el objetivo de construir visiones contra-hegemónicas que legitimasen la posición de China en el sistema internacional. ${ }^{19}$ En cambio, tanto el Partido Comunista Chino (PCCh) como la academia censuraban sistemáticamente todas aquellas teorías occidentales no marxistas por considerarlas herramientas teóricas al servicio del sistema capitalista.

\footnotetext{
${ }^{16}$ Ikeda, Josuke: “Japanese Vision of International Society: A Historical Exploration”, en Shimizu, Kosuke; Ikeda, Josuke; Kamino, Tomoya y Sato, Shiro (2008): Is There a Japanese IR? Seeking an Academic Bridge through Japan's History of International Relations, Otsu, Afrasian Centre for Peace and Development Studies, Ryukoku University, pp. 29-45.

${ }^{17}$ La escuela de Kyoto surgió en el periodo de entreguerras del siglo pasado y da nombre a un grupo de intelectuales establecidos en la Universidad Imperial de Kyoto. Se considera la escuela filosófica más importante de la historia intelectual de Japón, vid. Shimizu, Kosuke: "Materializing the "non-Western": two stories of Japanese Philosophers on Culture and Politics in the Inter-War Period", Cambridge Review of International Affairs, vol. 28, n $^{\circ} 1$ (julio 2014), pp. 3-20.

${ }_{18}$ La discusión sobre el nacimiento de la disciplina ha sido abordada, entre otros, por Qin en "Why is there no" op. cit.; Qin, Yaqing: "Development of International Relations theory in China: Progress through Debates", International Relations of the Asia-Pacific, vol. 11, no 2 (mayo 2011), pp. 231-257; Qin, Yaqing: "Development of International Relations Theory in China”, International Studies, vol. 46, no 1-2 (enero 2009), p. 185-201; Liang, Shoude: "Constructing an International Relations Theory with "Chinese Characteristics"”, Political Science, vol. 49, no 1 (julio 1997), pp. 23-39; Song, Xinning: "International Relations Theory-Building in China”, Political Science, vol. 49, n 1 (julio 1997), pp. 40-61; Song, Xinning: "Building International Relations Theory with Chinese Characteristics", Journal of Contemporary China, vol. 10, no 26 (febrero 2001), pp. 61-74; Ren, Xiao: “Lilun yu guoji guanxi lilun: yixie sikao' [Theory and IR Theory: Some Thoughts]", Chinese Journal of European Studies, n 4 (julio-agosto 2000), pp. 19-25; Wang, Jianwei: "International Relations Studies in China", Journal of East Asian Studies, vol. 2, n 1 (febrero 2002), pp. 69-87; Wang, Yiwei: "Between Science and Art: Questionable International Relations Theories", Japanese Journal of Political Science, vol. 8, no 2 (agosto 2007), pp. 191 y Lu, Peng: "Pre-1949 Chinese IR: an Occluded History", Australian Journal of International Affairs, vol. 68, n⿳0 2 (marzo 2014), pp. 133-155.

${ }^{19}$ Song "Building International Relations Theory", op. cit.
} 
A partir de la década de los ochenta, con la aparición de un nuevo horizonte económico, se inició un espacio institucional que facilitó la importación de las Ciencias Sociales occidentales. Como señala Liang, ${ }^{20}$ Deng Xiaoping manifestó que los estudios sobre las RRII, la Ciencia Política y las Ciencias Jurídicas necesitaban "recuperar el tiempo perdido". Como consecuencia de ello, en un intento de alejarse de la tradición marxista y el legado maoísta que había guiado a las Ciencias Sociales hasta aquel entonces, Deng incitó a los académicos a investigar todas aquellas teorías "rivales" provenientes de los Estados Unidos y Europa Occidental para poder aprender de ellas y extraer toda aquella información que beneficiase a una China que empezaba su período de reformas. Este período de distensión que experimentaron las Ciencias Sociales facilitó la llegada de traducciones de clásicos occidentales, y con ello se produjo el nacimiento de los estudios internacionales. ${ }^{21}$

Sin embargo, en contraposición a la narrativa tradicional, durante los años ochenta aparecieron dos visiones alternativas que empezaron a identificar el nacimiento de la disciplina en períodos diferentes. Mientras un grupo de teóricos la situaba en la década de los años veinte y coincidiendo con el gobierno de la República de China, un grupo más amplio de académicos lo ubicaba en un período indeterminado entre el establecimiento de la República Popular China (1949) y la apertura de China (1978). Esta disparidad de opiniones sobre el nacimiento de la disciplina nos sugiere que identificarla en uno u otro período conlleva connotaciones políticas e ideológicas diferentes. Mientras que aquellos que sitúan el surgimiento durante la década de los años veinte resaltan la relevancia del gobierno nacionalista del Guomintang, aquellos que sostienen que la disciplina nació entre 1949 y 1978 destacan el papel del PCCh como agente decisivo a la hora de asentar conocimiento.

Por lo que se refiere a los autores que apuestan por la primera visión, en su artículo Pre1949 Chinese IR: an occluded history ${ }^{22}$ Lu demostró como durante el gobierno de la República de China se habían creado ya los primeros estudios internacionales en algunos centros académicos como la Universidad de Beijing, la Universidad Nacional de Tsinghua o la Universidad de Saint John. La existencia de dichos estudios, además de cuestionar la narrativa tradicional, apuntala la idea de que su desarrollo fue evolutivo, es decir, un proceso que experimentó momentos de popularidad y períodos de abandono. De este modo, mientras que con la llegada de Mao al poder en 1949 hubo un interés en promover el estudio de la disciplina, durante el periodo que va desde los años cincuenta hasta finales de los setenta se dejaron de impartir las RRII y su estudio no reaparecería hasta la apertura económica. ${ }^{23}$

A pesar del análisis pormenorizado realizado por $\mathrm{Lu}$, la mayoría de los académicos chinos sigue negando que la disciplina existiese durante el gobierno de la República. Solamente un pequeño grupo de teóricos como $\operatorname{Chan}^{24}$ y Qin ${ }^{25}$ aceptan que su creación fue anterior a la apertura económica. En particular, Qin ha identificado el nacimiento de la disciplina en 1953, año a partir del cual las RRII experimentan un proceso de desarrollo en un sentido lakatosiano, ${ }^{26}$

\footnotetext{
${ }^{20}$ Liang "Constructing an International Relations", op. cit.

${ }^{21}$ Qin "Development of International Relations theory in China: Progress", op. cit.

${ }^{22} \mathrm{Lu}$, op. cit.

${ }^{23}$ Ibid., p. 152.

${ }^{24}$ Chan, Gerald: "International Studies in China: origins and development", Issues and Studies, vol. 33, $\mathrm{n}^{\mathrm{o}} 2$ (febrero 1997), pp. 40-64.

${ }^{25}$ Qin, "Why is there no", op. cit.

${ }^{26}$ Lakatos ha afirmado que la ciencia avanza a través de la creación de "programas de investigación" que compiten entre ellos. Para el autor, un programa está definido por un conjunto de teorías, hipótesis y reglas metodológicas que indican qué rutas debe seguir una investigación. Vid. Lakatos, Imre (1978): The Methodology of Scientific Research Programmes: Philosophical Papers Volume 1, Cambridge, Cambridge University Press. De forma similar, Qin, utilizando la metodología lakatosiana, sostiene que en los años previos a la apertura de China y a la llegada de la disciplina de las RRII durante los años ochenta no existía una teoría con características chinas, puesto
} 
es decir, los académicos chinos, a pesar de encontrarse aún en un estadio embrionario, se embarcaron en la creación de nuevos "programas de investigación" sobre estudios internacionales. El surgimiento de dichas teorías coincidió con la apertura por parte del gobierno chino tanto del Departamento de Estudios Diplomáticos de la Universidad Renmin de Beijing (1953), la primera institución que abordó cuestiones relacionadas con las RRII, como del Foreign Affairs College, un centro académico que ofrecía cursos de diplomacia a los cuadros del PCCh. La tarea principal de ambas instituciones era enseñar a los miembros de la futura élite gubernamental las distintas líneas políticas promovidas por el gobierno chino en materia de política exterior. En definitiva, para Qin, los procesos institucionales que ocurrieron durante la instauración de la República Popular China fueron cruciales para el nacimiento de la disciplina.

\subsection{La evolución teórica de las RRII en China: del marxismo a la "fiebre cultural"}

Como hemos apuntado, durante el período maoísta la hegemonía del marxismo-leninismo entre los académicos había imposibilitado la adopción de teorías que cuestionasen el sistema comunista. Ahora bien, con la apertura económica de Deng Xiaoping, la tendencia de una parte de la academia por pasar página del maoísmo y algunos de los episodios más trágicos como la Revolución Cultural permitió empezar a debatir el uso de teorías que hasta aquel momento habían estado prohibidas. Este cambio de paradigma generará un conflicto generacional entre los profesores más jóvenes o formados en el extranjero, ${ }^{27}$ que empezarán a rechazar las contribuciones académicas surgidas durante la China de Mao, y los académicos más veteranos, que seguirán defendiendo la existencia de un cuerpo teórico durante el maoísmo que identifican como esencial para el desarrollo posterior de la disciplina.

Más allá de estas diferencias generacionales e ideológicas, en este estudio consideramos que el marxismo y el maoísmo han contribuido en el desarrollo de la disciplina gracias a su cuestionamiento implícito del sistema de valores y normas de la sociedad internacional westfaliana. De esta forma, el marxismo sinizado por el pensamiento de Mao estableció las bases teóricas con las que cuestionar las ideas, normas y valores que apuntalan el orden global liberal euroamericano. La llegada de teorías poscoloniales, neoconfucianas y posmodernas durante la década de los noventa terminaron por complementar la tarea de la tradición marxista. ${ }^{28}$

Por otra parte, durante los primeros años de apertura económica, los académicos chinos buscaron respuestas a las contradicciones inherentes de un capitalismo en ciernes. ¿Cómo podía un Estado marxista mantener su legitimidad si su base ideológica colisionaba frontalmente con la realidad económica? Para solventar dichas contradicciones y dotar al Estado con una base ideológica coherente, los intelectuales chinos combinaron elementos del pensamiento político tradicional con el marxismo-leninismo y ciertos aspectos ontológicos del sistema westfaliano. Esta combinación de diferentes tradiciones de pensamiento político es la que inspirará la construcción de las "teorías con características chinas".

\footnotetext{
que no se crearon "programas de investigación científica" con estructuras definidas por un núcleo central y un "cinturón protector", por usar la expresión del filósofo. Vid. Qin, "Why is there no", op. cit., pp 314-317.

${ }^{27}$ A título de ejemplo, Qin, uno de los autores más relevantes de la academia china, obtuvo su doctorado en Ciencia Política en la Universidad de Missouri-Columbia.

${ }^{28}$ Los estudios poscoloniales suelen centrarse en criticar la modernidad euroamericana y algunas ideas que se originaron durante la Ilustración europea en el siglo XVIII, como el concepto de racionalidad. Para estos autores, la racionalidad sirvió como pretexto ideológico de las grandes potencias europeas para sus aventuras coloniales durante el siglo XIX. Para una lectura sobre dicho tema vid. Said, Edward W. (1979): Orientalism, Nueva York, First Vintage Books Edition. En la disciplina de las RRII también han surgido intentos de combinar los estudios poscoloniales con las RRII, vid. Ling, op. cit. y Seth, Sanjay (ed.) (2013): Postcolonial Theory and International Relations: a Critical Introduction, London, Routledge.
} 
Durante el periodo posmaoísta la evolución de las RRII se ha desarrollado en tres etapas. En la primera etapa (1980-1987) se produjeron una serie de debates sobre si China debía seguir siendo un Estado proletario revolucionario o si debía convertirse en un "Estado normal". ${ }^{29}$ En el ámbito teórico, se generó una revolución kuhniana en la que los paradigmas previos, como las tesis de Lenin, aunque no fueron rechazadas, sí fueron complementados o matizados. En otras disciplinas académicas, como la economía, el paradigma marxista fue parcialmente sustituido por teorías sociales provenientes de Occidente con la finalidad de facilitar la entrada de China al orden internacional liberal y que ésta se ajustase a las nuevas necesidades socioeconómicas y políticas. Un claro ejemplo de este cambio de paradigma lo podemos observar en los quince artículos publicados en la revista académica Política y Economía Mundial (世界经济与政治, shijie jingji yu zhengzhi) entre 1982 y 1983, en los que se cuestionaba si la teoría de Lenin sobre el imperialismo resultaba aún válida. ${ }^{30}$ En un mismo sentido, en el campo de los estudios culturales, la visita del crítico literario estadounidense Fredric Jameson a la Universidad de Beijing en 1985 contribuyó a la llegada de los estudios posmodernos. ${ }^{31}$ La aparición de sus estudios y de nuevas premisas teóricas y metodológicas fueron cruciales para el desarrollo de la teoría autóctona de RRII en China. El posmodernismo facilitó la posibilidad de trascender las grandes narrativas eurocéntricas construidas por la teoría de RRII euroamericana.

La segunda etapa (1987-2000) se caracterizó por la coexistencia de dos procesos paralelos: la llegada de las primeras traducciones de las obras clásicas euroamericanas de la las RRII y la voluntad de un grupo de académicos de crear una "teoría con características chinas". 32 Por lo que se refiere al primer proceso, a principios de la década de los noventa se empezaron a traducir al chino obras de autores realistas tales como Politics Among Nations de Hans Morgenthau (1990) o Theory of International Politics de Kenneth Waltz (1993). ${ }^{33}$ El profesor Yan Xuetong, ${ }^{34}$ quién como veremos en el siguiente apartado ha sido una figura clave en la academia china, tras su estancia académica en los Estados Unidos empezó a diseminar las tesis realistas entre los especialistas de su país. Como consecuencia de ello, la traducción de estos trabajos tuvo un gran impacto en la academia china y el realismo devino el segundo paradigma más popular entre los académicos chinos, tras el marxismo-leninista ortodoxo, que seguía manteniendo una posición de privilegio. ${ }^{35}$

La llegada de estas obras realistas coincidió con un período en el que tanto los académicos como los promotores de la política exterior china debían definir los objetivos internacionales de una China en transición. Ahora bien, mientras parte de la academia china estaba sumergida en una "fiebre cultural" traduciendo y diseminando las obras realistas de la disciplina, a finales de la década de los ochenta, empezaron a aparecer críticas a la incapacidad de la teoría euroamericana para interpretar de forma eficaz la política exterior china. Para algunos autores, como Liang ${ }^{36}$ la insuficiencia de la teoría euroamericana para analizar el caso

\footnotetext{
${ }^{29}$ Qin "Development of International Relations theory in China: Progress", op. cit., p. 236.

${ }^{30}$ Ibid. y Geeraerts, Gustaaf y Jing Men: "International Relations Theory in China", Global Society, vol. 15, nº 3 (mayo 2001), pp. 251-276.

${ }^{31}$ Dirlik, Arif y Zhang, Xudong: "Introduction: Postmodernism and China”, Boundary 2, vol. $24, \mathrm{n}^{\mathbf{o}} 3$ (otoño 1997), pp. 1-18.

${ }^{32}$ Entre ellos se encuentran el presidente del Foreign Affairs College, Liu Chun, y el director del Departamento de Investigación de Estudios Internacionales del consejo de Estado, Zhang Mingqian.

${ }^{33}$ Las fechas que aparecen junto las obras clásicas de RRII son los años en los que se publicaron las traducciones al chino, no el año de publicación en los Estados Unidos.

${ }^{34}$ Yan obtuvo su doctorado en la Universidad de California, Berkeley. Dicha experiencia le condujo a aceptar unos postulados positivistas que han ejercido una gran influencia en sus obras.

${ }^{35}$ Qin "Development of International Relations theory in China: Progress", op. cit., p. 238.

${ }^{36}$ Liang, Shoude: "Lun Guoji zhengzhixue de zhongguo tese [On Chinese characteristics of International Politics studies]", Guoji Zhengzhi Yanjiu, no 1 (enero-marzo 1994), pp. 15-21. y Liang "Constructing an International Relations", op. cit.
} 
chino requería la búsqueda de nuevos modelos que se aproximen a la política china desde una perspectiva autóctona.

En la primera conferencia en China sobre teoría de las RRII (上海全国际关理论研讨 会, shanghai quan guojiguan lilun yan taohui), celebrada en Shangai en $1987,{ }^{37}$ se apuntó por primera vez la necesidad de producir una "teoría de las RRII con características chinas". Algunos académicos y altos cuadros del partido afirmaron que las teorías euroamericanas y soviética no podían ser consideradas un modelo universalmente aceptado por lo que para salvaguardar los intereses nacionales de China y apuntalar los principios ideológicos del $\mathrm{PCCh}^{38}$ era necesario establecer una "teoría con características chinas". Ahora bien, para algunos críticos ${ }^{39}$ dicho concepto albergaba una fuerte carga ideológica y representaba una construcción teórica basada en un pensamiento político marxista-leninista y un confucianismo cuyo objetivo ulterior era legitimar y justificar la reemergencia de China y el liderazgo del PCCh. Más que aportar una visión plural a la disciplina, este proyecto teórico estaba intrínsecamente relacionado con la ideología del partido y representaba una reminiscencia del concepto de "socialismo con características chinas" de Deng. En definitiva, dichos autores denunciaban cómo la producción de teoría se limitaba a la reproducción de un proyecto político subyugado a los intereses del PCCh.

Durante la década de los noventa, coincidiendo con una etapa crecimiento económico ininterrumpido, el liberalismo experimentó su momento álgido y devino el paradigma dominante entre los académicos chinos. ${ }^{40}$ Durante este período se tradujeron al mandarín obras liberales como Power and Interdependence de Joseph Nye y Robert Keohane (1992) o After the Hegemony: Cooperation and Discord in the World Political Economy de Robert Keohane (2000). Tanto el liberalismo como el realismo se erigieron como las principales escuelas teóricas en la academia china y ello tuvo un hondo impacto en el diseño y confección de la política exterior del país. ${ }^{41}$ Además, este período de traducciones coincidió con un conjunto de debates que se sucedieron en el seno de la academia china sobre cuáles eran las principales amenazas para la seguridad nacional. A diferencia de las visiones realistas, los académicos liberales enfatizaron la importancia de las amenazas transnacionales derivadas de la pujante globalización liberal que se aceleró con el colapso de la Unión Soviética. ${ }^{42}$

La tercera y última etapa (2000- hasta la actualidad) se singulariza por la coexistencia de los tres paradigmas euroamericanos — realismo, liberalismo y constructivismo- con el

\footnotetext{
${ }^{37}$ Como explica Wang, más de 100 académicos chinos asistieron a esta conferencia con el objetivo de identificar el objeto de estudio y sistematizar los marcos teóricos de las RRII. Vid. Wang, Jianwei: "International Relations Studies in China", op. cit. Entre los académicos y funcionarios que asistieron a la conferencia se encontraban Wang Dao Han, antiguo profesor de la Universidad de Jiaotong de Shanghai; Huang Xiang, director del Consejo de Estado del Instituto de RRII; Liang Shoude, antiguo director del Departamento de RRII de la Universidad de Beijing; Ni Shixiong, profesor de RRII de la Universidad de Fudan de Shanghai; Jin Yingzhong, profesor del Centro de Investigación sobre Estrategia Internacional en Asia-Pacífico en Shanghai, y Zhang Mingqian, profesor de la Universidad de RRII en Beijing, cuya obra más relevante es: El gran despertar: Un estudio de la religión americana y las relaciones sociales 大觉醒 : 美国宗教与社会关系研究. Vid. Zhang, Mingqian (2001): Da juexing: meiguo zhongjiao yu shehui guanxi yanjiu. [El gran despertar: Un estudio de la religión americana y las relaciones sociales], Beijing, Shishi Chuban She.

${ }^{38}$ Liang "Constructing an International Relations”, op. cit. y Song "International Relations Theory-Building”, op. cit.

${ }^{39}$ Song "Building International Relations Theory", op. cit. y Callahan, William A.: "China and the Globalization of IR Theory: discussion of 'Building International Relations Theory with Chinese Characteristics"', Journal of Contemporary China, vol. 10, $\mathrm{n}^{\circ} 26$ (febrero 2001), pp. 75-88.

${ }^{40}$ Qin "Development of International Relations theory in China: Progress", op. cit., p. 240.

${ }^{41}$ Ibid., p. 243.

${ }^{42}$ Ibid., p. 242. y Deng, Yong: "The Chinese Conception of National Interests in International Relations", The China Quarterly, nº 154 (junio 1998), pp. 308-329.
} 
marxismo-leninista y, finalmente, la aparición de la Escuela china de las RRII (中国学派, Zhongguo xuepai). Esta última Escuela requiere un análisis más detallado por el impacto que desde entonces ha producido en la academia china y en la occidental.

\subsection{La aparición de la Escuela china de RRII}

El origen de la Escuela china de las RRII se remonta al año 2000 y el vocablo se ha usado desde entonces para designar a un grupo heterogéneo de autores críticos con las excesivas connotaciones ideológicas de la "teoría con características chinas". Como resultado de este espíritu contestatario, la Escuela china se ha confeccionado con elementos provenientes del confucianismo, el constructivismo, el liberalismo y los estudios poscoloniales. Además, se ha nutrido de los debates sobre las teorías no-occidentales que comenzaron a emerger en el corazón de la comunidad académica anglosajona a partir de finales de la década de los años noventa. Dichas discusiones abrieron un nuevo horizonte intelectual que facilitó el ascenso de prominentes intelectuales chinos que empezaron a concebir teorías de RRII construidas a través de la práctica política de China y sus tradiciones de pensamiento político.

Entre los primeros autores impulsores de la Escuela china destacan tres de los académicos que más activamente han contribuido en su formulación teórica: Mei Ran, ${ }^{43}$ Ren Xiao $^{44}$ y Wang Yiwei. ${ }^{45}$ Por una parte, $\operatorname{Ran}^{46}$ ha denunciado el carácter profundamente occidentalcéntrico de una disciplina cuya teoría no explica los fenómenos internacionales de una manera objetiva. Para Ran, la teoría es más bien una herramienta para defender y promover la agenda política de Occidente. Por ello, este autor plantea la necesidad de producir una teoría autóctona que explique la realidad política china. Por otra parte, $\operatorname{Ren}{ }^{47}$ ha sostenido que los académicos chinos deben aprender a reflexionar de una manera independiente y convertirse en "productores de conocimiento". En un mismo sentido, Wang Yiwei, ${ }^{48}$ desde el Instituto de RRII de la Universidad Renmin, ha identificado como la gran herencia cultural china ha facilitado la construcción de un vocabulario propio que, en última instancia, debe sustituir o complementar los términos analíticos occidentales. ${ }^{49}$ El planteamiento de Wang se ha convertido en uno de los ejes vertebradores de la Escuela china de RRII y, en los últimos quince años, se ha creado un léxico influenciado por el pensamiento tradicional chino y las prácticas sociales chinas que, como detallaremos a continuación, ha generado grandes debates tanto en China como en el exterior.

El interés por estudiar la Escuela china de RRII ha trascendido las fronteras del país y cada vez son más los autores occidentales que investigan esta corriente. ${ }^{50}$ Entre una parte de

\footnotetext{
${ }^{43}$ Mei Ran es profesor asociado de RRII en el departamento de estudios internacionales de la Universidad de Beijing en Beijing.

${ }^{44}$ Ren Xiao es profesor de RRII y director del Centro de estudios de política exterior china en la Universidad de Fudan en Shanghai.

${ }^{45}$ Es importante destacar que todos ellos han formado parte de la nomenclatura china y de su élite intelectual. Es decir, su conocimiento no sólo procede del ámbito académico sino también de la práctica política como diplomáticos o asesores del gobierno chino.

${ }^{46}$ Ran, Mei: "Gai bu gai you guoji zhengzhi lilun de zhongguo xuepai - jian ping meiguo de guoji zhengzhi lilun" [Should There be a Chinese School of IR Theory? Remarks on American Theories of International Politics]", Guoji Zhengzhi Yanjiu, n 1 (enero-marzo 2000), p. 63.

${ }^{47}$ Ren "Lilun yu guoji guanxi", op. cit.

${ }^{48}$ Wang Yiwei es profesor y director del Instituto de RRII de la Universidad Renmin en Beijing.

${ }^{49}$ Li, Mao: "China Should Speak its Own Language, Scholars Say at IR Meeting”, Chinese Social Science Today, 25 de julio de 2013, en http://www.csstoday.com/Item/254.aspx

${ }^{50}$ Chan, Gerald (1999): Chinese Perspectives on International Relations: a Framework for Analysis, New York, St. Martin's Press; Paltiel, Jeremy T.: "Constructing Global Order with Chinese Characteristics: Yan Xuetong and the Pre-Qin Response to International Anarchy", The Chinese Journal of International Politics, vol. 4, $\mathrm{n}^{\circ} 4$ (diciembre 2011), pp. 375-403; Zhang, Feng (2012): "Debating the "Chinese Theory of International Relations": Toward a New Stage in China's International studies”, en Dallmayr, Fred y Zhao, Tingyang (eds.): In
} 
estos teóricos existe la idea generalizada que el nacimiento de la Escuela china se explica fundamentalmente por factores de tipo estructural. ${ }^{51}$ De tal suerte, entienden que la innovación teórica de los académicos chinos está directamente vinculada con el aumento de las capacidades materiales de un país, y consecuentemente este enorme poder económico y militar ha impulsado la creación de conocimiento autóctono de las RRII. Sin embargo, esta visión tiende a ignorar dos factores cruciales: las dinámicas internas de la disciplina y la interacción entre la disciplina china y la euroamericana.

Por un lado, en China han existido innovaciones teóricas incluso en periodos en los que el Estado chino no gozaba de unas capacidades materiales como las actuales. Un claro ejemplo lo encontramos en la Teoría de los tres mundos (三个世界的理论, Sange shijie de lilun) que se originó durante la época maoísta. Dicha teoría, a pesar de su orientación de tipo práctico, nació con una clara vocación por entender el sistema internacional en el que China se estaba insertando. Por otro lado, la innovación teórica es también el resultado de los intercambios culturales entre la academia china y la euroamericana. Precisamente las dinámicas de la globalización liberal han permitido un incremento de las interacciones entre la academia china y las demás academias del planeta, no solamente enriqueciéndola, sino también generando múltiples aproximaciones, a veces contradictorias las unas con las otras.

Un claro ejemplo de las contradicciones existentes se puede encontrar en la convivencia de enfoques teóricos aparentemente antagónicos como el liberalismo, el tradicionalismo y el marxismo en la academia china. Dichas contradicciones se explican fundamentalmente por dos procesos. Por un lado, los académicos chinos se han convertido en agentes de la importación de ideas liberales que $a$ priori resultan incompatibles con los valores anti-liberales del gobierno chino que pretende promover. Por otro lado, a pesar del buen recibimiento de la Escuela china en la comunidad académica euroamericana, la tendencia de los académicos chinos a inspirarse en el pensamiento tradicional para crear teorías netamente autóctonas y originales ha dificultado

Contemporary Chinese Political Thought. Debates and Perspectives. Lexington, University of Kentucky Press, pp. 67-87; Qin, Yaqing: "Cultura y pensamiento global: una Teoría china de las Relaciones Internacionales", Documentos CIDOB Asia, no 28 (septiembre 2013), pp. 67-90; Cunningham-Cross, Linsay (2014): In search of a Chinese School: Ghostly encounters with the parochial/global discipline of international relations, University of Manchester [tesis doctoral]; Noesselt, Nele: "Revisiting the Debate on Constructing a Theory of International Relations with Chinese Characteristics", The China Quarterly, no 222 (junio 2015), pp. 430-448; Kim, Hun Joon: "Will IR theory with Chinese Characteristics be a Powerful Alternative?", The Chinese Journal of International Politics, vol. 9, no 1 (marzo 2016), pp. 59-79; Zhang, Yongjin, y Chang, Teng-Chi (eds.) (2016): Constructing a Chinese School of International Relations: Ongoing Debates and Sociological Realities, London, Routledge; Lu, Peng: "Chinese IR Sino-centrism tradition and its influence on the Chinese School Movement", The Pacific Review, (April 2018), pp. 1-18; Do, Thuy T.: “China's rise and the 'Chinese dream' in international relations theory”, Global Change, Peace \& Security, vol. 27, nº 1 (Febrero 2015), pp. 21-38; Kristensen, Peter Marcus y Zhang Yongjin: "The curious case of 'Schools' of IR: From the sociology of the geopolitics of knowledge", The Chinese Journal of International Politics, vol. 10, $\mathrm{n}^{\circ} 4$ (Invierno 2017), pp. 429-454; Zhang, Chunman: "How to merge Western theories and Chinese indigenous theories to study Chinese politics", Journal of Chinese Political Science, vol. 22, n² (Junio 2017), pp. 283-294; Acharya, Amitav: "Theorising the international relations of Asia: necessity or indulgence? Some Reflections". The Pacific Review, vol. 30, n6 (Noviembre 2017), pp. 816-828; Li, Nan y Xie, Peng: "Guoji guanxi "zhongguo xuepai" de jiangou ji qi xiandai xing fansi [The construction of the "Chinese School” in International Relations and its reflection on modernity]", Journal of Shanxi Datong University (Social Science), vol. 33, no 1 (febrero 2019), pp. 15-19; Sun, Jisheng: "gaige kaifang yilai zhongguo guoji guanxi lilun fazhan huayu shijian yu chuangxin [Chinese IR theory development since China's reform and opening up: discourse, practice and innovation]", Shijie jingji yu zhengzhi, no 8 (junio 2018), pp. 4-29; Ni, Chang-wen: "guanyu guoji guanxi xue "san da fanshi" de fansi — yi bijiao zhengzhi xue he lishi shehui xue jiangou "zhongguo xuepai" [Reflections on the "Three theories" of International Relations - constructing the "Chinese School" in the light of comparative politics and historical sociology]", Jiangnan Shehui Xueyuan Xuebao, vol. 19, nº 2 (junio 2017), pp. 49-54 y Yan, Dexue: "Xijiping waijiao sixiang yu zhongguo tese guoji guanxi lilun goujian [Xi Jinping's thought on diplomacy and the construction of IR theories]", Dongbei Ya Luntan, n 1 (febrero 2019), pp.3-20.

${ }^{51}$ Moure, op. cit.; Noesselt, op. cit. y Zhang et al., op. cit. 
la homologación de la teoría china de RRII bajo la etiqueta "moderna" y "científica" que disfruta la teoría de RRII euroamericana. Por todo ello podemos afirmar que la Escuela china se encuentra en una fase de construcción y de mejora teórica constante. En el siguiente apartado analizaremos con más detalle la evolución reciente de la escuela.

\subsection{La evolución de la Escuela china: ¿múltiples proyectos?}

Durante los últimos quince años, el interés por analizar la Escuela china ha aumentado considerablemente, especialmente después de que algunos académicos manifestasen su voluntad de producir teoría de RRII a partir de elementos culturales chinos. A pesar de que la denominación de escuela en singular podría implicar la existencia de un proyecto teórico de naturaleza monolítica, la realidad es que abarca tres construcciones teóricas con diferentes cosmovisiones, reflejando una clara apuesta por el pluralismo teórico. ${ }^{52}$ Estas tres contribuciones teóricas están representadas por la Teoría Tianxia de Zhao Tingyang, el Realismo Moral de Yan Xuetong y la "Teoría de la Relacionalidad constructivista" de Qin Yaqing. ${ }^{53}$

Por lo que se refiere al primer caso, Zhao Tingyang, filósofo neo-confuciano de la Academia china de Ciencias Sociales, ${ }^{54}$ ha desarrollado la teoría Tianxia (天下, "bajo el cielo"), un concepto inspirado en la dinastía Zhou (1122 a.n.e y 249 a.n.e.) que se sustentaba en un sistema de gobernanza en cuya cúspide se situaba China -el país del centro- y alrededor de ella gravitaban los demás países. Ahora bien, a pesar de la asimetría de poder entre el centro y los países periféricos, este sistema no se basaba en el uso de la fuerza, sino en el establecimiento de acuerdos que beneficiaban a todas las naciones. Ello resultó en un sistema de gobernanza regional en el que la potencia más poderosa renunciaba a comportarse de forma hegemónica y los demás países, aceptaban esta asimetría de poder a cambio de mantener un cierto grado de autonomía política.

Aplicado en un contexto como el actual, para $\mathrm{Zhao}^{55}$ este proyecto teórico debe inspirar el establecimiento de un nuevo orden gobernado por una institución que sea universalmente aceptada por los miembros de la comunidad internacional y cuyo objetivo sea superar un sistema westfaliano que el autor considera como un "mundo fallido sumergido en el caos". Para Qin $^{56}$ el sistema Tianxia está justificado en términos políticos siempre y cuando la gobernanza global resultante pueda beneficiar de forma armónica a todos los países. De este modo, la naturaleza epistemológica de la teoría Tianxia es de cariz pospositivista y se basa en una excepcionalidad cultural china que pretende, a la vez, universalizar prácticas políticas

\footnotetext{
${ }^{52}$ El concepto de Escuela china de RRII se encuentra fuertemente inspirado por la "Escuela inglesa de RRII". Ahora bien, mientras en la Escuela inglesa se analiza la creación de una "sociedad internacional" basada en el principio de soberanía, la diplomacia y el derecho internacional, en el caso del proyecto intelectual de China se pretende analizar el "ascenso pacífico de China". Por otro lado, el concepto de "teoría" se refiere a la existencia de paradigmas, una serie de premisas que sirven como marcos de referencia para poder entender los temas mencionados anteriormente.

${ }^{53}$ A pesar de que el académico taiwanés Shih Chih-Yu no suele estar incluido en la Escuela china de RRII, conviene destacar que su teoría del "Equilibrio de relaciones" se asemeja al proyecto teórico de Qin Yaqing. La teoría del "Equilibrio de relaciones" de Huang y Shih busca la reciprocidad y la armonía a través de la promoción de relaciones recíprocas a largo plazo con las que evitar la incertidumbre derivada de la anarquía sistémica. Si el "Equilibrio de poder" promueve los intereses egoístas, el "Equilibrio de relaciones" busca la autocontención. Vid. Kim, op. cit. p. 72 y Huang, Chiung-Chiu y Shih, Chih-yu (2014): Harmonious intervention: China's quest for relational security, Farnham, Ashgate.

${ }^{54}$ Zhao, Tingyang: "Rethinking Empire from a Chinese Concept "All-under-Heaven" (Tian-xia)", Social Identities, vol. 12, n 1 (enero 2006), pp. 29-41; Zhao, Tingyang: "A Political World Philosophy in terms of Allunder-heaven (Tianxia)”, Diogenes, no 221 (febrero 2009), pp. 5-18 y Zhao, Tingyang (2018): Tianxia tout sous un même ciel. L'ordre du monde dans le passé et pour le futur, Paris, Les Éditions du Cerf.

${ }^{55}$ Zhao "Rethinking Empire from a", op. cit. p. 36

${ }^{56}$ Qin "Cultura y pensamiento global”, op. cit. pp. 3-4.
} 
tradicionales chinas. Ahora bien, a diferencia de las teorías que veremos en los siguientes apartados, la innovación teórica de Zhao presenta elementos culturales que sólo se generan en sociedades confucianas como la china.

El concepto de Tianxia se ha convertido en una de las propuestas teóricas más desarrolladas y exitosas de la academia china, y ello se explica por dos factores. Por un lado, el concepto ha servido como punto de partida para la construcción de teorías de las RRII inspiradas en el pensamiento tradicional y en las prácticas sociales chinas. Por otro lado, el concepto de Tianxia ha trascendido la esfera académica y los medios de comunicación y ha marcado la agenda y los discursos políticos de algunos dirigentes del PCCh como Hu Jintao o Xi Jinping. En el año 2005, el entonces presidente Hu Jintao realizó un discurso ante la Asamblea General de las Naciones Unidas en el que hizo múltiples referencias a uno de los conceptos que derivan de la teoría del Tianxia: la idea de “mundo armonioso" (和谐世界, hexie shijie)..$^{57}$

Ahora bien, a pesar de la vocación universalista de la teoría Tianxia como alternativa al orden liberal, este proyecto ha despertado una profunda desconfianza entre aquellos que lo identifican como un pretexto ideológico creado por intelectuales cercanos al aparato del Estado chino para justificar el ascenso del país como gran potencia del sistema internacional. Como ha afirmado uno de los grandes críticos occidentales a la propuesta de Zhao, William Callahan, ${ }^{58}$ dicho proyecto conlleva la imposición de un sistema imperial chino basado en una gobernanza de tipo jerárquico que pondrá en peligro el orden liberal occidental.

El segundo proyecto está representado por la Escuela de Tsinghua ${ }^{59}$ y su principal teórico es Yan Xuetong. Yan ${ }^{60}$ ha estudiado exhaustivamente el pensamiento de los intelectuales chinos anteriores a la dinastía Qin (221-206 a.n.e.) - la época de los Reinos Combatientes - y ha establecido categorías conceptuales inspiradas en el pensamiento chino tradicional que se asemejan a los principales paradigmas de la teoría euroamericana —realismo, liberalismo y constructivismo— ${ }^{61}$. La adopción de dichas categorías le sirve a Yan para estudiar el pensamiento chino tradicional y demostrar la universalidad del pensamiento chino.

\footnotetext{
${ }^{57}$ La idea de "mundo armonioso" hace referencia a un ideal de orden internacional pacífico que se originó en la antigua China. Diversos pensadores tradicionales como Confucio, Mencio o la escuela taoísta desarrollaron dicho concepto. A finales del siglo XX, este fue recuperado por las elites del PCCh para legitimar y justificar el auge pacífico de China. Para un análisis sobre el uso del concepto vid. Hu, Jintao: "Build towards a Harmonious World of Lasting Peace and Common Prosperity", en Discurso ante la cumbre de las Naciones Unidas en New York (septiembre 2005) http://www.un.org/webcast/summit2005/statements15/china050915eng.pdf; Sheng, Ding: "To build a "harmonious world": China's soft power wielding in the global south", Journal of Chinese Political Science, vol. 13, n 2 (agosto 2008), pp. 193-213.

${ }^{58}$ Callahan, William A.: "Chinese Visions of World Order: Post-hegemonic or a New Hegemony?", International Studies Review, vol. 10, no 4 (diciembre 2008), pp. 749-761.

${ }^{59}$ El enfoque de la Escuela de Tsinghua nació en el departamento de RRII de la Universidad de Tsinghua en Beijing en la década de los noventa. El profesor Yan Xuetong, después de su experiencia en la academia estadounidense, decidió promover el método científico positivista y los análisis cuantitativos en el estudio de la historia de las relaciones internacionales de China y el pensamiento internacional chino. Para Yan, las Ciencias Sociales en China deben estar guiadas por el método científico occidental de testeo de hipótesis, análisis causales, objetividad y verificabilidad. Para un análisis sobre su obra vid. Zhang, Feng: "The Tsinghua Approach and the Inception of Chinese Theories of International Relations", The Chinese Journal of International Politics, vol. 5, $\mathrm{n}^{\circ} 1$ (marzo 2012), pp. 73-102. y Cunningham-Cross, Linsay: "Using the past to (re)write the future: Yan Xuetong, pre-Qin thought and the China's rise to power", China Information, vol. 26, no 2 (junio 2012), pp. $219-233$.

${ }^{60}$ Yan, Xuetong; Bell, Daniel A. (ed.); Sun, Zhe (ed.) y Ryden, Edmund (trad.) (2011): Ancient Chinese Thought, Modern Chinese Power, Princeton, Princeton University Press.

${ }^{61}$ Por poner un ejemplo, Yan identifica el pensamiento legalista de Hanfeizi dentro de la corriente realista.
} 
La principal innovación teórica de $\mathrm{Yan}^{62}$ es lo que él ha denominado Realismo Moral, un concepto que pretende dar una respuesta alternativa al ascenso de China y al futuro del orden mundial combinando elementos tanto del realismo como de la moralidad confuciana. Aunque a ojos de un teórico occidental puede resultar paradójico, para Yan el realismo debe ir acompañado de una dimensión moral de la política internacional para perseguir un doble objetivo. Por una parte, esta moralidad no solamente justifica el liderazgo político de un Estado que está en ascenso, sino que le permite influir en la creación de nuevas normas internacionales. Por otra parte, facilita el desarrollo de estrategias de poder blando gracias a las cuales un Estado actúa moralmente de forma correcta para mejorar la imagen y percepción que tienen los demás países sobre él. En definitiva, el objetivo final de esta estrategia es que los países con los que interactúa China terminen por tejer alianzas militares, económicas o culturales con Beijing.

Para analizar los tipos de Estados según su legitimidad moral, Yan utiliza la clasificación que propuso el pensador Xunzi en el siglo III a.n.e sobre los Reinos Combatientes. Según el filósofo, los Estados de la antigua china cimentaron su legitimidad a través de tres modelos de gobernanza. En primer lugar, los gobernantes podían dirigir sus Estados a través de una autoridad humana (王, Wang) que simbolizaba el arquetipo de gobierno ideal. La legitimidad estaba universalmente aceptada y se producía una relación de respeto entre los gobernantes y los gobernados y viceversa. El segundo modelo era la hegemonía (霸, $B a$ ), un tipo de gobernanza en que el uso de la fuerza implicaba una legitimidad más débil. A pesar de que no era un tipo ideal, se mantenía un orden político que los gobernados estaban dispuestos a aceptar. El último modelo era la tiranía (强, Qiang), un gobierno que empleaba la violencia hacia los gobernados de forma más intensa que en los dos casos anteriores. Para Yan, la tiranía era el modelo de gobernanza más deleznable puesto que implicaba un nivel de legitimidad débil que ponía en riesgo el orden político. Según Xunzi, la importancia de estos tres modelos reside en sus fuentes de legitimidad moral, ya que en última instancia éstas pueden alterar las normas y valores que sostienen el sistema internacional. Dicho en otras palabras, el sistema internacional puede variar dependiendo de la legitimidad moral de los Estados que lo componen.

Considerando la vocación universal de la categorización de Xunzi, con su Realismo Moral, y partiendo desde postulados científicos positivista, Yan afirma que las teorías, los paradigmas, ${ }^{63}$ los programas de investigación ${ }^{64}$ o las "tradiciones de investigación" 65 occidentales ofrecen las herramientas analíticas suficientes para comprender las RRII en China. Por ello, a pesar de que paradójicamente el trabajo de Yan suele estar incluido en la Escuela china de RRII por su contribución teórica desde una perspectiva autóctona, el mismo autor sostiene que no es factible ni necesario establecer una Escuela china. Para Yan cualquier teoría que siga el método científico positivista debe mantener una vocación universal.

\footnotetext{
${ }^{62}$ Yan, Xuetong: "Political Leadership and Power Redistribution", The Chinese Journal of International Politics, vol. $9, \mathrm{n}^{\circ} 1$ (marzo 2016), pp. 1-26.

${ }^{63}$ Kuhn, Thomas S. (1962): The Structure of Scientific Revolutions, Chicago, University of Chicago Press.

${ }^{64}$ Lakatos, op. cit.

${ }^{65}$ Laudan, Larry (1978): Progress and its Problems: Towards a Theory of Scientific Growth, Los Angeles, University of California Press.
} 
Por último, Qin Yaqing, ${ }^{66}$ una de las principales figuras que han diseminado el constructivismo en China, propone la teoría de la "Relacionalidad constructivista" ${ }^{67}$ un modelo híbrido que combina conceptos relativos a las prácticas culturales chinas como el guanxi ${ }^{68}$ y la dialéctica del yin-yang con elementos del paradigma constructivista euroamericano. Para Qin ${ }^{69}$ el mundo se debe estudiar a partir de las relaciones sociales entre los agentes que lo componen. Por ello, para comprender el comportamiento de los actores no debemos analizar sus características o cualidades inherentes, sino sus relaciones con otros actores — normalmente Estados- . Sin interacciones sociales no se puede crear una identidad que es lo que termina por influir el comportamiento, los intereses o los valores de dicho actor. Todo ello supone una ruptura con la tradición westfaliana de las RRII que tiende a estudiar el comportamiento de los actores a partir de sus unas características pre-configuradas.

Otro aspecto crucial de su obra es su particular interpretación del concepto de poder. El pensamiento filosófico euroamericano ha entendido que el poder es el resultado de una relación asimétrica entre actores con diferentes capacidades materiales. Sin embargo, para Qin, ${ }^{70}$ el poder se desarrolla siguiendo una red de relaciones muy similar a la que se produce en el seno de las familias confucianas. Según este modelo de análisis, el poder no es tanto el resultado de unas determinadas capacidades materiales, sino que es el producto del significado simbólico que la sociedad confuciana confiere a las relaciones familiares. Por lo tanto, aunque Qin no niega el aspecto material del poder, la esencia del mismo reside en su constitución simbólica.

En lo referente a la dimensión epistemológica de su teoría, Qin acepta el método científico occidental y su pretendido universalismo, pero construye su teoría con elementos propios de la cultura china como la dialéctica del yin-yang o el concepto de guanxi. Basándose en una definición lakatosiana sobre cómo se deben construir las teorías, Qin ${ }^{71}$ afirma que éstas deben convertirse en "programas de investigación" semejantes al paradigma realista, liberal o constructivista. Sin embargo, su concepción de la naturaleza del cambio social y los procesos entre actores es netamente de inspiración taoísta.

Las innovaciones teóricas planteadas por los tres teóricos analizados han sido desarrolladas a partir de diferentes puntos de partida. Por una parte, la diferencia entre las tesis de Qin y Yan reside en que el primero utiliza la cultura china como fuente ontológica para producir una teoría que complemente los paradigmas tradicionales euroamericanos, mientras que Yan entiende la cultura china como partida para validar el universalismo de los paradigmas tradicionales occidentales. En cambio, la proposición de Zhao, inspirada netamente en el pensamiento político tradicional, rechaza que la teoría euroamericana sea universalmente

\footnotetext{
${ }^{66}$ Qin Yaqing es presidente y profesor de RRII en la China Foreign Affairs University (CFAU). Asimismo, es canciller de la Academia de diplomacia china y vicepresidente ejecutivo de las Asociación de estudios internacionales en China (CNAIS). Para un análisis de su obra vid. Qin, Yaqing: "Relationality and processual construction: bringing Chinese ideas into international relations theory", Social Sciences in China, vol. $30, \mathrm{n}^{\circ} 3$ (agosto 2009), pp. 5-20 y Qin, Yaqing (2018): A relational theory of World politics, Cambridge, Cambridge University Press.

${ }^{67}$ La teoría de la "Relacionalidad constructivista" que propone Qin incorpora y conceptualiza dos ideas chinas: los procesos y las relaciones. Los procesos sociales son la fuente ontológica de dicha teoría. Vid. Ibid. En cambio, la red de relaciones en la sociedad internacional ayuda a los Estados a formar sus identidades.

${ }^{68}$ El guanxi es una práctica habitual en la sociedad china que consiste en establecimiento y desarrollo de relaciones personales gracias al cual se compone una red de contactos que en última instancia promueven la cooperación entre los individuos que forman parte de dicho grupo social. Para una lectura sobre el concepto de guanxi vid. Chang, En-Chung; Chou Ting-Jui; Huang, Chungming y Wang, Xun: "The Categories, Rules, and Demonstrations of Guanxi in Chinese Society", Journal of Business-to-Business Marketing, vol. 23, n 4 (octubre 2016), pp. 311325.

${ }^{69}$ Qin "Relationality and processual construction”, op.cit., p.14.

${ }^{70}$ Qin "Relationality and processual construction”, op.cit., pp.16-17.

${ }^{71}$ Qin "Why is there no", op. cit.
} 
válida. Consecuentemente, el filósofo neo-confuciano propone un nuevo modelo político que sustituya el sistema internacional westfaliano puesto que en él reside el origen de todos los problemas políticos, económicos y sociales del mundo actual.

A pesar de las diferencias ontológicas y epistemológicas de los proyectos teóricos analizados, estos presentan dos características comunes. Por un lado, buscan una comprensión holística sobre la reemergencia de China desde su apertura económica y coinciden en apuntar que dicho ascenso será pacífico y armonioso. Por otro lado, intentan ofrecer soluciones teóricas ante uno de los grandes temores de la élite del PCCh: la histórica "trampa de Tucídides". Basándose en los estudios del historiador griego, la trampa apunta la idea que el estallido de un conflicto bélico entre un poder hegemónico en declive - Estados Unidos- y una potencia emergente - China - se debe a las tensiones estructurales y a la desconfianza mutua surgidas de la incapacidad de ambas potencias para acomodarse al nuevo contexto. En la actualidad, uno de los objetivos primordiales del presidente Xi Jinping es demostrar a la comunidad internacional que el ascenso de China será pacífico y que, por lo tanto, el país será capaz de evitar la trampa de Tucídides. Un claro ejemplo de tal esfuerzo, lo podemos encontrar en su pensamiento que analizamos en la siguiente sección.

\subsection{El pensamiento de Xi Jinping y la Escuela china de RRII}

El estudio del pensamiento de Xi Jinping (习近平新时代中国特色社会主义思想，Xi Jinping xinshidai zhongguo tese shehui huizhuyi xixiang) ${ }^{72}$ resulta clave para interpretar la relación entre la Escuela china de RRII y el auge de China como potencia. Los esfuerzos de la Escuela china en desarrollar una visión normativa que guíe el ascenso pacífico de China se han manifestado en los discursos del presidente Xi. Específicamente, dicha conexión se puede encontrar en uno de los conceptos que el Presidente Xi destacó durante su discurso ante el XIX Congreso del Partido Comunista de 2017: “la comunidad de un destino común” (人类命运共 同体, renlei mingyun gongtongti). Se trata de un concepto normativo del pensamiento diplomático chino contemporáneo que ha sido recuperado por el gobierno de Xi para dotar a China de herramientas con las que contribuir a la gobernanza global y a la creación de un orden internacional. ${ }^{73}$ Esta expresión apareció por primera vez durante el discurso del ex-presidente Hu Jintao ante el XVIII Congreso Nacional del Partido Comunista chino en noviembre de $2012^{74}$ y en la que hacía referencia a la posibilidad de construir un orden internacional complementario al liberal basado en la coexistencia pacífica y en los valores del pensamiento tradicional chino. Sin embargo, el Presidente Xi Jinping no solamente adoptó dicha idea como

\footnotetext{
${ }^{72}$ Para una lectura sobre los debates en torno al pensamiento de Xi Jinping en los principales medios de comunicación y la comunidad académica vid. Mardell, Jacob: The "'Community of Common Destiny' in Xi Jinping's New Era", The Diplomat, 25 de octubre de 2017, en https://thediplomat.com/2017/10/the-communityof-common-destiny-in-xi-jinpings-new-era/; Qiong, Liu: "Xijinping: Rang mingyun gongtongti yishi zhai zhoubian guojia luodi Shenggen [Xi Jinping: Let the community of common destiny take root in neighboring countries], Xinhua Net, 25 de octubre de 2013, en http://www.xinhuanet.com//politics/201310/25/c_117878944.htm ; Peters, Michael A.: "The Chinese Dream: Xi Jinping thought on socialism with Chinese characteristics for a new era", Educational Philosophy and Theory, vol. 49, no 14 (noviembre 2017), pp. 12991304; Smith, Stephen N.: "Community of common destiny: China's "new assertiveness" and the changing Asian order", International Journal, vol. 73, n 3 (setiembre 2018), pp. 449-463 y Buckley, Chris: "Xi Jinping thought explained: a new ideology for a new era", New York Times, 26 de febrero de 2018, en https://www.nytimes.com/2018/02/26/world/asia/xi-jinping-thought-explained-a-new-ideology-for-a-new-

era.html; Goh, Sui Noi: "19th Party Congress: Xi Jinping outlines new thought on socialism with Chinese traits", The Strait Times, 28 de octubre de 2017, en https://www.straitstimes.com/asia/east-asia/19th-party-congress-xijinping-outlines-new-thought-on-socialism-with-chinese-traits

${ }^{73}$ Zhang, Denghua: “The concept of 'Community of Common Destiny' in China's Diplomacy: Meaning, Motives and Implications”, Asia \& the Pacific Policy Studies, vol. 5, no 2 (enero 2018), pp. 196-207.

${ }^{74}$ Jintao, Hu: "Report of Hu Jintao to the 18th CPC National Congress", China.org.cn, 16 de noviembre de 2012, en http://www.china.org.cn/china/18th_cpc_congress/2012-11/16/content_27137540_11.htm
} 
propia, sino que además la convirtió en uno de los pilares fundamentales de la actual política doméstica y exterior china, asociándola explícitamente al destino político de China. ${ }^{75}$

Durante los dos últimos años del mandato de Xi, el desarrollo de una "comunidad de destino común" se ha convertido en la prioridad del gobierno chino y piedra angular que vertebra y cohesiona la política exterior y doméstica china. La voluntad de Xi de acelerar la creación de dicho orden internacional se debe a dos variables. Por un lado, el estado actual del contexto internacional y regional es cada vez más volátil y ello puede afectar negativamente al auge de China. Por lo tanto, la implementación de dicho orden internacional complementario proporciona un anclaje en el que China pueda desarrollar sus capacidades materiales y normativas en el ámbito doméstico e internacional de una manera más calmada. Por otro lado, como resultado de la existente volatilidad internacional, China entiende que debe adoptar un papel más activo en la gobernanza global para poder eludir aquellos efectos negativos de la nueva realidad internacional. Así pues, la creación de dicho orden internacional facilita la implementación de un orden complementario al liberal donde China puede mantener un rol más independiente y de mayor liderazgo.

La implementación de una "comunidad de destino común" no sólo requiere la creación de instituciones, acuerdos militares o de recursos financieros, sino que precisa de un conjunto de ideas que guíen este proyecto y que contribuyan a la concepción de una sociedad internacional complementaria con características chinas. Ha sido en la fase de creación del concepto, donde la Escuela china de RRII ha jugado un papel fundamental. Por un lado, ésta ha proporcionado un conjunto de herramientas discursivas al gobierno chino que han legitimado el proyecto. Por otro lado, la Escuela china ha asentado aquellos elementos normativos con características chinas que contribuyan a la regulación del orden internacional. Como consecuencia de esta diferenciación cultural que destaca la singularidad normativa del proyecto, la "comunidad de destino común" pretende establecerse como orden complementario y autónomo.

En el discurso ante el XIX Congreso Nacional del Partido Comunista de 2017, Xi formuló los aspectos normativos de la "comunidad de destino común" en el que sintetizó los tres proyectos de la Escuela china - la teoría del Tianxia, el Realismo Moral y la teoría de la "Relacionalidad constructivista". Xi presentó una visión normativa del orden internacional, fuertemente inspirada en la teoría del Tianxia en la que animaba a compartir la responsabilidad para solucionar los futuros retos comunes. ${ }^{76}$ Esta propuesta representa una alternativa a un orden westfaliano caracterizado por Estados que mantienen sus intereses egoístas y que son incapaces de divisar cualquier tipo de "destino común". ${ }^{77}$

Para Xi, la creación de dicha comunidad no sólo depende del desarrollo pacífico de China sino también del fomento de unas relaciones con los demás actores que estén basadas en el beneficio mutuo. ${ }^{78}$ Para conseguir este objetivo, la obra de Qin Yaqing es crucial. Como hemos visto anteriormente, éste promueve unas relaciones de cooperación - yin-yang-entre el orden internacional chino y el liberal. Esta visión facilita la coexistencia de ambos órdenes donde el mundo liberal se puede beneficiar del mundo chino y viceversa. Es decir, dicha visión normativa busca trascender la lógica realista de suma cero que cada vez más está rigiendo las relaciones internacionales contemporáneas.

\footnotetext{
${ }^{75}$ Secure a Decisive Victory in Building a Moderately Prosperous Society in All Respects and Strive for the Great Success of Socialism with Chinese Characteristics for a New Era, en http://www.xinhuanet.com/english/download/Xi_Jinping's_report_at_19th_CPC_National_Congress.pdf

${ }^{76}$ Ibid.

77 Ibid.

${ }^{78}$ Ibid.
} 
Respecto a la regulación de dichas relaciones y su impacto en la gobernanza global, Xi hizo alusión a “la defensa de la justicia” (秉持正确义利观, Bingchi zhengqueyiliguan), ${ }^{79}$ referencia implícita a una de las premisas del Realismo Moral de Yan Xuetong. Como hemos visto anteriormente, Yan sostiene que la predicación con el ejemplo y la defensa de valores como la justicia pueden favorecer un estilo de gobernanza capaz de generar una hegemonía positiva. Esto se debe a que ambos elementos éticos y morales se pueden emplear como herramientas de poder blando para atraer a otros Estados o instituciones internacionales a la órbita del orden chino. Por lo tanto, la referencia de Xi a la "defensa de la justicia" ${ }^{80}$ no es tanto una proclama en un sentido liberal, sino más bien una declaración de intenciones. En definitiva, Xi aspira a crear un modelo de gobernanza complementario al orden occidental que se asemeje al de autoridad humana (王, wang) mencionado por Yan Xuetong.

En este sentido, la influencia de la Escuela china en el pensamiento de Xi cabe interpretarse como la necesidad de encontrar un discurso que justifique la construcción de dicho orden internacional complementario y que facilite una mayor autonomía política en una China en ascenso. Sin embargo, un estudio pormenorizado del pensamiento y las ideas de Xi indican que para el gobierno chino la creación de este orden es una opción complementaria al orden internacional existente y no un reto al mismo. Por lo tanto, en estas líneas cuestionamos aquellos estudios que describen con un cierto desasosiego la irrupción de una China revisionista. En definitiva, entendemos que la reciente asertividad de China no es consecuencia de su voluntad de convertirse en un nuevo hegemón, sino más bien de su deseo de construir una sociedad internacional complementaria que le permita satisfacer sus necesidades políticas y socioeconómicas.

\section{La disciplina de las RRII en Japón: una producción teórica marcada por su política exterior}

En las últimas décadas el interés por las RRII en Japón ha aumentado, no solamente entre los académicos japoneses, sino también entre los teóricos occidentales. Ahora bien, en lugar de centrarse en producir teoría de las RRII, la academia japonesa ha adoptado una perspectiva de estudios de área y los escasos estudios teóricos existentes han sido construidos a partir de las tradiciones occidentales. ${ }^{81}$ A pesar de la existencia de una gran variedad de aproximaciones paradigmáticas entre los estudiosos japoneses, para entender cómo los académicos han participado en dichas discusiones conviene centrar el estudio en tres cuestiones cruciales: cuándo se integraron las diferentes tradiciones occidentales de las Ciencias Sociales en la academia japonesa; ${ }^{82}$ cómo se han desarrollado los debates paradigmáticos de las RRII en el Japón de posguerra (1945-1990); y, finalmente, de qué forma han repercutido ciertas singularidades histórico-sociales en la evolución de la disciplina y en la creación de teorías autóctonas.

\footnotetext{
79 Ibid.

${ }^{80}$ Ibid.

${ }^{81}$ Corrêa Malafaia, Thiago: "Japanese International Relations: An assessment of the 1971-2011 period", Brazilian Political Science Review, vol. 10, $\mathrm{n}^{\circ} 1$ (abril 2016), p. 8. y Do, Thuy T. (2016): The practices of knowledge claims: Reflections from the drive toward constructing "East Asian International Relations Theory". The Australian National University [tesis doctoral], p. 132.

${ }^{82}$ Se ha optado por mantener el concepto "tradición" tal como lo emplea Inoguchi e Inoguchi y Bacon. Vid. Inoguchi, Takashi (1989): "The Study of International Relations", en The Study of International Relations: The State of the Art, New York, Palgrave Macmillan, pp. 250-264; Inoguchi, Takashi y Bacon Paul: "The study of International Relations in Japan: towards a more International Discipline", International Relations of the AsiaPacific, vol. 1, no 1 (enero 2001), pp. 1-20 y Inoguchi, Takashi: "Are there any Theories of International Relations in Japan?" International Relations of the Asia-Pacific, vol. 7, no 3 (septiembre 2007), pp. 369-390.
} 
En primer lugar, para Inoguchi y Bacon ${ }^{83}$ el surgimiento y la evolución de la disciplina en Japón desde el siglo XIX hasta la actualidad son el resultado de tradiciones de pensamiento que representan formas diferentes de aprehender la realidad. Ahora bien, a diferencia de la ya clásica clasificación de Wight et. al. ${ }^{84}$ para ambos autores han existido en Japón, cuatro grandes tradiciones en las Ciencias Sociales que han convivido sin excesiva interacción entre ellas: la teoría general del Estado (Staatslehre) que se incorporó en Japón tras la restauración Meiji (1868), el marxismo (a partir de los años veinte del siglo XX), el historicismo (a partir de $1945)^{85}$ y el positivismo cuyo auge se produjo a partir de los años setenta. ${ }^{86}$ Por lo tanto, sería más adecuado entenderlas como corrientes de pensamiento que llegan a Japón y modifican las aproximaciones ontológicas y epistemológicas de los académicos que estudian la historia de la diplomacia. ${ }^{87}$

En segundo lugar, si bien el estudio de las tradiciones de pensamiento nos permite entender el progreso de la disciplina en Japón, para comprender cómo se ha teorizado en este ámbito desde la Segunda Guerra Mundial resulta necesario analizar la aparición y la omisión de algunos de los debates paradigmáticos en la academia japonesa. Sobre el primer debate, existe una tendencia a establecer una analogía entre el primer gran debate (idealismo versus realismo) de los años veinte con el discusión sobre la revisión del Tratado de Seguridad entre Japón y los Estados Unidos (1960) ${ }^{88}$ que emergió en la última mitad de la década de 1950 y se intensificó en los años sesenta y setenta. ${ }^{89}$ En Japón, la discusión no se centraba en la naturaleza pacifista del país, cuya condición era asumida mayoritariamente por los responsables políticos y por el conjunto de la sociedad, sino en identificar cuál era la mejor forma de expresar dicho pacifismo. Más bien, el debate aludía a la aparición de dos posiciones antagónicas ante la revisión del Tratado de Seguridad: aquellos que aceptaban a los Estados Unidos como el garante de la seguridad japonesa (realismo), y los que se posicionaban claramente en contra del Tratado (idealismo). En este segundo grupo heterogéneo convivían posiciones intermedias, desde autores que denunciaban la contradicción entre el artículo 9 de la Constitución japonesa ${ }^{90}$ y la presencia de fuerzas militares estadounidenses en territorio japonés, hasta los autores que rechazaban los nuevos compromisos que estaba asumiendo Japón en el contexto de Guerra Fría.

\footnotetext{
${ }^{83}$ Inoguchi et al., op. cit. e Inoguchi “Are there any Theories”, op. cit.

${ }^{84}$ Wight entendía las tradiciones de pensamiento político como preexistentes a la disciplina de las RRII y las clasificó principalmente según los objetos de estudio. Vid. Wight et al. op. cit. Sobre las tradiciones de pensamiento según Wight vid. la nota al pie 3.

${ }^{85}$ Shimizu destaca la relación entre el historicismo y los estudios de área en la academia japonesa de las RRII. Sobre esta cuestión vid. Shimizu, Kosuke: "The genealogy of culturalist international relations in Japan and its implications for post-Western discourse", All Azimuth, vol. 7, no 1 (enero 2018), p. 133. https://doi.org/https://dx.doi.org/10.20991/allazimuth.326972

${ }^{86}$ En la actualidad, el Staatslehre, el historicismo y el positivismo son las tradiciones preponderantes en Japón, puesto que la tradición marxista ha perdido gran parte de su seguimiento desde la década de los años sesenta. vid. Inoguchi "Are there any Theories", op. cit. y Yamamoto, Kazuya: "International Relations Studies and Theories in Japan: a Trajectory Shaped by War, Pacifism, and Globalization", International Relations of the Asia-Pacific, vol. 11, no 2 (mayo 2011).

87 Inoguchi "The Study of International Relations", op. cit.

${ }^{88}$ Inoguchi, en cambio, argumenta que el motivo central del debate entre idealistas y realistas no era la revisión del Tratado de Seguridad entre Japón y los Estados Unidos, sino sobre la idoneidad del Tratado de San Francisco (1951). Vid. Inoguchi “Are there any Theories”, op. cit., p. 376.

${ }^{89}$ Chen, Ching-Chang: "The Im/Possibility of Building Indigenous Theories in a Hegemonic Discipline: The Case of Japanese International Relations", Asian Perspective, vol. 36, nº 3 (julio-septiembre 2012), p. 469 y Yamamoto, op. cit., pp. 263-265.

${ }^{90}$ El artículo 9 de la Constitución de Japón señala la renuncia a la amenaza y al uso de la fuerza para resolver las controversias y prohíbe el mantenimiento de fuerzas militares. No obstante, la interpretación de dicho artículo ha permitido que Japón cuente con fuerzas militares orientadas a la defensa nacional, las Fuerzas de Autodefensa.
} 
Sin embargo, ha sido la inexistencia del segundo gran debate de carácter epistemológico de los años cincuenta y sesenta lo que explica que no se haya impuesto una única metodología para analizar las RRII y que la tradición positivista no haya devenido dominante en Japón, como sí ocurrió en Occidente. La ausencia de dicho debate se interpreta por el estrecho vínculo entre las RRII y las tradiciones del Staatslehre y el historicismo, aproximaciones metodológicas que incorporan una considerable herencia del tradicionalismo. Así, las teorías japonesas de RRII, más que construirse a partir de proposiciones positivistas, se edificaron sobre la base de un tradicionalismo metodológico menos dependiente de hipótesis y comprobaciones empíricas.

Respecto al tercer debate, no se ha producido un consenso sobre su surgimiento. Mientras algunos autores como Yamamoto defienden su existencia, otros, como Inoguchi, niegan que el debate se haya iniciado. ${ }^{91}$ Esta falta de acuerdo sobre la existencia del debate interparadigmático es el resultado de dos fenómenos. Por un lado, la eclosión de autores que empezaron a estudiar las instituciones internacionales y los actores no estatales durante los años setenta y, por otro lado, la carencia de un diálogo real entre dichos autores y aquéllos que se valían de las teorías clásicas de las RRII. Además, si analizamos la situación reciente del cuarto gran debate, podemos afirmar que en Japón no se ha producido el debate entre racionalistas y pospositivistas. Ello se debe a que numerosos autores japoneses, si bien seguían una metodología menos positivista, identificaron sus estudios como reflectivistas desde antes de la aparición del debate en Occidente. ${ }^{92}$

Finalmente, sobre la última gran cuestión de cariz tanto ontológica como epistemológica, en 2007 Acharya y Buzan se interrogaron sobre si existía algún tipo de teoría de las RRII no occidental en su clásico "Why is there no non-Western international relations theory?"93 publicado en la revista International Relations of the Asia Pacific. Para algunos autores como Ikeda, ${ }^{94}$ Inoguchi, ${ }^{95}$ Kamino, ${ }^{96}$ Kobayashi $^{97}$ u Ong ${ }^{98}$ existe una producción de conocimiento de RRII japonesa con peculiaridades histórico-sociales distintas respecto a la producción occidental. ${ }^{99}$ Do $^{100}$ va más allá y sostiene que la academia japonesa ha recibido aportaciones principalmente de la escuela filosófica de Kyoto.

En los siguientes apartados estudiaremos las principales contribuciones teóricas que se han producido en Japón. En las décadas previas a la Segunda Guerra Mundial, en Japón se elaboraron teorías fundamentadas en las diferencias histórico-sociales entre Japón y Occidente - la teorización de la Esfera de Coprosperidad de la Gran Asia Oriental y el modelo de los

\footnotetext{
${ }^{91}$ La discusión entre Yamamoto e Inoguchi sobre si existió el tercer debate en Japón puede derivarse de cómo ellos entienden este debate. Por una parte, Yamamoto considera al tercer debate como el debate interparadigmático que oponía el estatocentrismo y el internacionalismo. Inoguchi, en cambio, entiende el tercer debate como el debate neo-neo. Vid. Ibid. e Inoguchi "Are there any Theories", op. cit.

${ }^{92}$ Inoguchi "Are there any Theories", op. cit., pp. 376-377.

${ }^{93}$ Acharya et al. "Why is there no non-Western international relations theory? An introduction", op. cit.

${ }^{94}$ Ikeda op. cit.

${ }^{95}$ Inoguchi "Are there any Theories", op. cit.

${ }^{96}$ Kamino, Tomoya: The Twenty Years' Crisis, 1919-1939: An Introduction of the Study of International Relations in Japan”, en Shimizu, Kosuke; Ikeda, Josuke; Kamino, Tomoya y Sato, Shiro (2008): Is There a Japanese IR? Seeking an Academic Bridge through Japan's History of International Relations, Otsu, Afrasian Centre for Peace and Development Studies, Ryukoku University, pp. 5-28.

${ }^{97}$ Kobayashi, Hiroharu (2003): "Royama Masamichi’s Perception of International Order from the 1920s to 1930s and the Concept of the East Asian Community", en Nationalism and Internationalism in Imperial Japan: Autonomy, Asian Brotherhood, ¿or World Citizenship?, New York, Routledge, pp. 135-168.

${ }^{98}$ Ong, Graham Gerard: "Building an IR Theory with 'Japanese Characteristics': Nishida Kitaro and 'Emptiness'”, Millennium, vol. 33, no 1 (enero 2004), pp. 35-58.

${ }^{99}$ Esta percepción no es generalizada y Corrêa Malafaia niega que las contribuciones de la academia japonesa puedan considerarse teorías debido a su naturaleza esencialmente filosófica, más que teórica, y por su falta de rigor metodológico. Vid. Corrêa Malafaia op. cit. pp. 9-10.
}

${ }^{100}$ Do op. cit. pp. 148-149. 
gansos voladores-. ${ }^{101}$ Tras la derrota japonesa en la Segunda Guerra Mundial, los objetivos de la academia japonesa cambiaron y, como veremos en el siguiente apartado, gran parte de los teóricos empezaron a preguntarse por la idoneidad de las decisiones tomadas por los líderes japoneses antes y durante la guerra.

\subsection{La Esfera de Coprosperidad de la Gran Asia Oriental y el modelo de los "gansos voladores"}

El precursor del estudio de las teorías no-occidentales en Japón, el politólogo Takeshi Inoguchi ${ }^{102}$ ha apuntado la idea que la academia japonesa no ha producido paradigmas que expliquen el funcionamiento del sistema internacional, sino que más bien se ha centrado en generar teorías de alcance medio para el caso concreto japonés. Acuñadas por Robert Merton, ${ }^{103}$ las teorías de alcance medio son una aproximación teórica de la sociología con un valor explicativo limitado y que versan sobre fenómenos concretos de la realidad. Dichas teorías se encuentran en un punto intermedio entre aquellas teorías alejadas de la realidad observable y aquellas afirmaciones demasiado concretas. ${ }^{104}$ Es decir, ni pretenden explicar toda la realidad social, ni tampoco representan proposiciones particulares no sistematizadas. Por todo ello, las teorías de alcance medio nos permiten diferenciar entre los paradigmas y las teorías que los constituyen. Si aplicamos el esquema mertoniano, en el presente trabajo entendemos que la producción teórica en Japón se encuentra asociada con dos teorías de rango medio: el desarrollo teórico de la política de la Esfera de Coprosperidad de la Gran Asia Oriental y el modelo de los "gansos voladores". 105

Autores como Inoguchi ${ }^{106}$ y Shimizu ${ }^{107}$ señalan que el primer desarrollo teórico, auspiciado por varios filósofos de la escuela de Kyoto, ${ }^{108}$ se formuló en relación con el concepto de la Esfera de Coprosperidad de la Gran Asia Oriental (大東亜共栄圈, Dai Tōa Kyōeiken). Dicho concepto fue anunciado por el gabinete del Príncipe Konoe en agosto de 1940 y representaba una evolución de la idea de Nuevo Orden de Asia Oriental (東亜新秩序, Tōa Shinchitsujo) formulado en 1938 para justificar la actuación de Japón en el noreste asiático. Si bien la idea inherente en ambos conceptos es la liberación de los países asiáticos de la opresión

\footnotetext{
${ }^{101}$ Corrêa Malafaia señala el modelo Kanban de Toyota como una de las contribuciones de la academia japonesa de los años 1930. Vid. Corrêa Malafaia, op. cit. p. 4. No obstante, este es principalmente un modelo de gestión empresarial, no una aportación a las RRII.

102 Inoguchi "Are there any Theories", op. cit.

${ }^{103}$ Merton, Robert K. (1968): Social Theory and Social Structure, New York, Free Press.

${ }^{104}$ Ibid. p. 39.

${ }^{105}$ El modelo de los "gansos voladores" adopta tal nombre por el paralelismo entre el desarrollo de los Estados y la formación de vuelo de los gansos. La formación de vuelo de los gansos se caracteriza por estar liderada por una de estas aves, mientras que las demás la siguen en forma de "V".

106 Inoguchi "Are there any Theories", op. cit.

${ }^{107}$ Shimizu, Kosuke: “Culture and International Relations: Why Do Japan's IR Focus on the Third Dimension?”, en Shimizu, Kosuke; Ikeda, Josuke; Kamino, Tomoya y Sato, Shiro (2008): Is There a Japanese IR? Seeking an Academic Bridge through Japan's History of International Relations, Otsu, Afrasian Centre for Peace and Development Studies, Ryukoku University, pp. 69-83.

${ }^{108}$ Para ahondar en las bases filosóficas de la escuela de Kyoto y la Esfera de Coprosperidad de la Gran Asia Oriental vid. Goto-Jones, Christopher S. (2005): Political Philosophy in Japan: Nishida, the Kyoto School and Co-prosperity, London, Routledge. Colegrove ha profundizado sobre los fundamentos teóricos de las políticas expansionistas japonesas y la evolución de la política exterior japonesa en los primeros años de la Guerra del Pacífico. Vid. Colegrove, Kenneth: "The New Order in East Asia", The Far Eastern Quarterly, vol. 1, n 1 (noviembre 1941), p. 5. Por último, para conocer en detalle la evolución de la historia diplomática japonesa del siglo XX, especialmente durante en los años treinta y cuarenta, vid. la guía bibliográfica de Asada, Sadao (1988): Japan and the World, 1853-1952: A Bibliographic Guide to Recent Scholarship in Japanese Foreign Relations, New York, Columbia University Press.
} 
colonial occidental y la cooperación entre los pueblos asiáticos, en la práctica servía para guiar una política imperialista japonesa y su consecuente expansión militar. ${ }^{109}$

La Esfera de Coprosperidad de la Gran Asia Oriental ofrecía una visión alternativa al orden internacional del período de entreguerras, cuya base jurídica se había fundamentado en los preceptos del Tratado de Versalles (1919) y el pacto de Kellogg-Briand (1928). El sistema internacional occidental que se estableció tras la Primera Guerra Mundial no consiguió satisfacer las expectativas y necesidades de los diferentes Estados de la región del este asiático. Como consecuencia, las élites japonesas comenzaron a cuestionar el orden internacional, la idea westfaliana de Estados soberanos y la dominación imperialista. ${ }^{10}$ Con todo, el fundamento meta-teórico de gran parte de estas críticas seguía siendo la diferenciación entre Oriente y Occidente, inherente también en la retórica occidental.

El concepto de Esfera de Coprosperidad de la Gran Asia Oriental que guió la política nacional y justificó la dominación militar hasta 1945 debe diferenciarse de algunos intentos teóricos de la época, entre los cuales destaca la teoría de la "East Asian Cooperative Community” (東亜協同体, Tōa Kyōdōtai) de Rōyama Masamichi y su Grupo de Investigación Showa (昭和研究会, Shōwa Kenkyūkai), del cual Rōyama formaba parte. ${ }^{111}$ Las bases teóricas de Rōyama, a diferencia de la política expansionista oficial, ${ }^{112}$ incorporaban elementos de respeto a la diversidad cultural, de fomento del desarrollo, de cooperación económica, de mejora de la vida de las personas y de autonomía de las naciones en Asia. ${ }^{113}$ Un proceso similar ocurrió con las aportaciones filosóficas de Nishida Kitaro y un gran número de filósofos de la Escuela de Kyoto, que fueron incorporadas en el discurso del gobierno imperial para justificar la expansión japonesa. ${ }^{114}$ La excesiva abstracción teórica de su obra era lo suficiente maleable para que sus ideas se incorporarsen en el discurso oficial. Además, la confrontación entre Oriente-Occidente, tema de estudio de la Escuela de Kyoto, desvió la atención de sus miembros, incapaces de centrarse en la devastación que un Imperio japonés ávido de colonias estaba generando. ${ }^{115}$ No obstante, algunos autores de la escuela de Kyoto, como Tosaka Jun, prestaron

\footnotetext{
${ }^{109}$ Colegrove, op. cit.

${ }^{110}$ Sobre cómo la Esfera de Coprosperidad de la Gran Asia Oriental se formuló como una iniciativa para superar la modernidad Occidental vid. Tosa, Hiroyuki (2018): "The Pitfalls in the Project of Overcoming Western Modernity: Rethinking the Lineage of Japanese Historical Revisionism", en Modern Japanese Political Thought and International Relations, London, Rowman \& Littlefield International, pp. 111-128.

${ }^{111}$ Rōyama, aunque no formaba parte de la escuela de Kyoto, tuvo un papel central en el Grupo de Investigación Showa. A este grupo generalmente se le atribuye el papel de cerebro del gabinete del Príncipe Konoe. Ahora bien, aunque existe una clara implicación del Grupo de Investigación Showa en el aparato imperialista, resulta igualmente cierto que afloraron diferencias entre la alineación de los miembros del grupo y las políticas finalmente adoptadas. Sobre esta cuestión vid. Kobayashi, op. cit. y Kamino, op. cit.

112 Aún no existe consenso sobre si algunos académicos teóricos como Nishida Kitaro, Tabata Shigejiro, Yanaihara Tadao o Yasui Kaoru secundaban la militarización y expansión del imperialismo japonés. Sobre esta cuestión vid. Arisaka, Yoko: “The Nishida Enigma: 'The Principle of the New World Order'”, Monumenta Nipponica, vol. 51, $\mathrm{n}^{\circ} 1$ (verano 1996), p. 81; Ikeda, op. cit., Nakano, Ryoko: “Pre-History' of International Relations in Japan: Yanaihara Tadao's Dual Perspective of Empire”, Millennium, vol. 35, no 2 (marzo 2007), pp. 301-319. y Nakano, Ryoko (2018): "Rethinking the Liberal/Pluralist Vision of Japan's Colonial Studies", en Modern Japanese Political Thought and International Relations, London, Rowman \& Littlefield International, pp. 111-128.

${ }^{113}$ Vid. Kobayashi, op. cit., p. 156 y Kamino, op. cit., pp. 38-39.

${ }^{114}$ Nishida es considerado el creador de la escuela filosófica de Kyoto. Las aportaciones filosófico-normativas de Nishida en la formación identitaria de Japón han llevado a Inoguchi a etiquetarlo como un "constructivista innato" o un "proto-constructivista". Vid. Inoguchi, Are there any Theories", op. cit. p. 370.

115 Shimizu, Kosuke: "Nishida Kitaro and Japan's interwar foreign policy: War involvement and culturalist political discourse", International Relations of the Asia-Pacific, vol. 11, nº 1 (enero, 2011), pp. 174-175 y Shimizu, Kosuke: "Materializing the 'non-Western': two stories of Japanese philosophers on culture and politics in the interwar period", Cambridge Review of International Affairs, vol. 28, nº 1 (2015), p. 15.
} 
más atención al impacto de las políticas sobre los ciudadanos y manifestaron una postura crítica sobre la deriva imperialista de Japón y sobre la confrontación geopolítica de la época. ${ }^{116}$

Ikeda Josuke, ${ }^{117}$ uno de los autores que con más profundidad ha abordado el estudio de las de teorías autóctonas de RRII en el Japón imperial, clasifica a los autores japoneses anteriores a 1945 en cuatro grandes grupos -idealistas, kelsenianos, cosmopolitas y panasiáticos-. Asimismo, relaciona sus temáticas de investigación acerca de la sociedad internacional sobre la base de tres ideas: solidaridad, autoridad y autonomía. Estas ideas se asemejan a los temas que Inoguchi ${ }^{118}$ entiende implícitos en la formulación teórica de la Esfera de Coprosperidad de la Gran Asia Oriental, a saber, la igualdad, el antimonopolio de Occidente y la solidaridad en Asia Oriental. Específicamente, para Ikeda, ${ }^{119}$ la producción japonesa se diferencia de Occidente en la formulación del concepto de autonomía en el que Japón se sitúa a medio camino entre el mundo de los colonizadores y de los colonizados - in-between-ness - . En concreto, esta concepción de la autonomía se articuló políticamente a través de formulación de la Esfera de Coprosperidad de la Gran Asia Oriental. La percepción de Japón como potencia in-between tiene parte de su fundamento teórico en las aportaciones filosóficas de Nishida $-\mathrm{y}$ otros miembros de la escuela de Kyoto- que identificaban a Japón como un agente que evitaba significarse y se situaba en una posición de ventaja como puente entre Occidente y Oriente. De este modo, Japón podría devenir una sociedad que absorbiera elementos culturales con múltiples orígenes. $^{120}$

Esta idea de in-between-ness también devendrá un elemento relevante en el modelo de los “gansos voladores” (雁行形態論, Gankō Keitairon o flying geese). El concepto, propuesto por Akamatsu Kaname en los años treinta y popularizado en la década de los sesenta del siglo pasado, representa una teorización sobre el comercio y el desarrollo económico de lo que Inoguchi ${ }^{121}$ ha descrito como ejemplo del patrón de interacción de Japón con Asia y el mundo. Según Akamastu, los "gansos voladores" hacen referencia a un proceso de aprendizaje por parte de los Estados menos desarrollados en Asia Oriental para que puedan lograr un alto nivel de industrialización que debía terminar generando libertad y bienestar al conjunto de la humanidad.

Más allá del discurso oficial, el modelo de los "gansos voladores" se empleó como parte de la propaganda de la Esfera de Coprosperidad de la Gran Asia Oriental que pretendía justificar la expansión japonesa hacia los países asiáticos. Bajo dicho modelo se estableció un sistema comercial desigual gracias al cual Japón compraba a sus colonias productos de menor gama como metales, comida o algodón, y les vendía productos sofisticados como fertilizantes, químicos o bienes del capital. ${ }^{122}$ No obstante, Akamatsu formulaba dicho modelo situando a los países occidentales como líderes y, a los asiáticos, como "países seguidores", siendo Japón el país más avanzado de este último grupo. ${ }^{123}$ Ahora bien, Akamatsu defendía que la no injerencia

\footnotetext{
${ }^{116}$ Ibid. p. 15.

${ }^{117}$ Ikeda, op. cit.

${ }^{118}$ Inoguchi "Are there any Theories", op. cit., p. 385.

${ }^{119}$ Ikeda, op. cit.

${ }^{120}$ Shimizu "Nishida Kitaro and Japan's", op. cit., 177.

121 Inoguchi "Are there any Theories", op. cit., p. 385.

${ }^{122}$ Corrêa Malafaia op. cit. p. 7.

${ }^{123}$ Posteriores estudios situaron a Japón como una economía líder. Vid. Kasahara, Shigehisa: “The Flying Geese Paradigm: A Critical Study of Its Application to East Asian Regional Development”, UNCTAD Discussion, $\mathrm{n}^{\circ} 169$ (abril 2004), p. 11. Para conocer en mayor detalle el modelo de los "gansos voladores" y su evolución vid. Akamatsu, Kaname: "A Historical Pattern of Economic Growth in Developing countries", The Developing Economies, vol. 1, (agosto 1962), pp. 3-25; Kojima, Kiyoshi: "The "Flying Geese" Model of Asian Economic Development: Origin, Theoretical Extensions, and Regional Policy Implications", Journal of Asian Economics, vol. 11, no 4 (otoño 2000), pp. 375-401; Korhonen, Pekka: "The Theory of the Flying Geese Pattern of Development and Its Interpretations", Journal of Peace Research, vol. 31, nº 1 (febrero 1994), pp. 93-108 y Ozawa,
} 
de las potencias extranjeras era crucial para alcanzar el desarrollo económico. De esta manera, el modelo de los "gansos voladores" incorpora las particularidades de la academia japonesa propuestas por Ikeda: la defensa de la autonomía nacional y el estatus de in-between-ness.

Los modelos de RRII en Japón no solo incorporan elementos meta-teóricos, ${ }^{124}$ sino también un cierto desarrollo teórico que se concreta en los dos ejemplos de teorías de rango medio que acabamos de analizar. En definitiva, durante el periodo de entreguerras en el que Japón anhelaba compararse con las potencias occidentales. Como se argumentará en el siguiente apartado, esta producción teórica autóctona contrasta con la escasez teórica de la academia japonesa en el periodo posterior a la Segunda Guerra Mundial.

\subsection{La evolución de las RRII en Japón desde la Segunda Guerra Mundial}

Para entender la reciente evolución de las RRII, resulta de suma utilidad el estudio de Inoguchi y Bacon ${ }^{125}$ en el que se identifican tres preguntas centrales que han guiado a la disciplina desde el final de la Segunda Guerra Mundial: ¿qué se hizo mal para que Japón terminará inmerso en la Guerra del Pacífico?, ¿qué tipos de acuerdos internacionales son deseables para garantizar la paz? y ¿por qué debe aún mejorar tanto la política exterior japonesa? La primera cuestión plantea cuáles fueron las circunstancias que llevaron a Japón a la guerra. La segunda, enmarcada en el primer gran debate de las RRII en Japón, concierne a la negociación de los acuerdos tras la contienda, la posición de Japón en la Guerra Fría y la pertinencia del Tratado de Seguridad con los Estados Unidos. En definitiva, estas primeras preguntas deben entenderse en relación con la posición de Japón como país vencido y en su condición de aliado de los Estados Unidos en una región fragmentada por las lealtades antagónicas impuestas por la lógica bipolar.

Este trasfondo histórico explica tanto la aproximación realista de la academia japonesa al concepto de seguridad como, al mismo tiempo, el interés en el estudio del pacifismo. Como consecuencia, los teóricos japoneses se aproximaron a las RRII partiendo de una epistemología tradicionalista hasta la década de los años setenta, momento en el que se empezaron a incorporar las diferentes metodologías y objetos de estudio provenientes del positivismo imperante en la academia norteamericana. ${ }^{126}$ La evolución de la disciplina en los Estados Unidos, las tensiones comerciales que Japón mantenía con los países desarrollados y las crisis del petróleo de los setenta ampliaron la agenda de temas de investigación sobre economía, comercio transnacional o finanzas internacionales.

En este contexto, apareció la tercera de las cuestiones de la academia japonesa sobre el porqué debía mejorar la política exterior del país. Esta tercera pregunta ha suscitado estudios comparativos entre la política exterior japonesa y la de otros Estados desarrollados e investigaciones sobre cómo mejorar la eficiencia de la política exterior japonesa. Desde entonces, la academia se ha ido aproximando a la epistemología occidental mainstream, incorporando progresivamente las metodologías positivistas.

Asimismo, durante la década de los setenta del siglo pasado, en Japón se formuló un concepto que tuvo un impacto significativo en la evolución teórica de la disciplina: la seguridad

\footnotetext{
Terutomo: "The "Hidden" Side of the "Flying-Geese" Catch-up Model: Japan's Dirigiste Institutional Setup and a Deepening Financial Morass", Journal of Asian Economics, vol. 12, no 4 (invierno 2001), pp. 471-491.

${ }^{124}$ La idea que, en Asia, los pueblos sometidos al imperialismo occidental debían seguir un camino autónomo con solidaridad entre los pueblos no genera, por sí sola, una teoría, pero puede ser la base para producir teorías como la East Asian Cooperative Community. De forma muy similar, la idea de in-between-ness, que para Ikeda es la fuente las teorías japonesas de las RRII, responde más a un fundamento meta-teórico que a una teoría por sí misma. Otros autores a los que se les atribuye cierto fundamento meta-teórico son Tabata Shigejiro, quien replanteó la igualdad de Estados soberanos o Hirano Yoshitaro, quien situó el regionalismo por encima de la soberanía nacional. Inoguchi “Are there any Theories”, op. cit.

${ }^{125}$ Inoguchi et al., op. cit.

${ }^{126}$ Yamamoto, op. cit., p. 274.
} 
comprehensiva. Dicho concepto aborda aquellos aspectos no puramente militares de la seguridad nacional tales como la seguridad alimentaria, la seguridad energética o la gestión de desastres naturales. Este constructo teórico, más allá de su impacto en la disciplina, destaca por dos factores: en primer lugar, se trata de una idea propiamente japonesa que se popularizó durante la década de los ochenta y que ha devenido la piedra angular del discurso de la política de seguridad japonesa de las últimas décadas. En segundo lugar, a pesar de ser un concepto teórico, desde un inicio se ha introducido en la praxis de la política exterior. Por ello, Yamamoto $^{127}$ argumenta que el desarrollo del concepto de seguridad a escala global cada vez se parece más al pacifismo japonés. Esta asimilación no se debe a un cambio sustancial en la academia japonesa, sino a la generalización del concepto de seguridad humana, ${ }^{128}$ el cual también incorpora asuntos no militares como la seguridad alimentaria, el medio ambiente o las comunicaciones.

Debido a la incorporación de metodologías y objetos de estudio occidentales, los académicos japoneses han tendido a interpretar los eventos desde el punto de vista de los diferentes paradigmas occidentales de las RRII. Aun así, la academia japonesa se ha diversificado incorporando tanto las teorías críticas como el constructivismo y enfatizando el rol normativo de las ideas para definir los intereses, los comportamientos y la estructura del sistema internacional. ${ }^{129}$

A diferencia de lo que hemos analizado con el caso chino, Do ${ }^{130}$ destaca que la academia japonesa de las RRII no ha desarrollado una escuela nacional de las RRII propia, sino que sus miembros se han enzarzado en discusiones sobre si existen teorías no-occidentales en Japón. Esta falta de incentivos por establecer una escuela nacional se explica por el uso indebido que el gobierno japonés hizo de la Escuela de Kyoto para justificar el imperialismo, por el declive relativo que está experimentando Japón en la actualidad y por una identidad ambigua basada en una dualidad entre Occidente y Oriente. Si bien en la actualidad los académicos más jóvenes, muchos de ellos instruidos en Occidente, muestran un mayor interés mayor por las aproximaciones teóricas, las discusiones sobre la existencia de teorías no-occidentales en Japón han impactado de forma limitada en una academia japonesa que mantiene su preferencia por los estudios empíricos e históricos. ${ }^{131}$

En la actualidad, y fruto de las diferencias metodológicas y ontológicas, en Japón existen grupos de académicos que investigan asuntos que son menos habituales en Occidente: la noproliferación de armas nucleares, las operaciones de Naciones Unidas para el mantenimiento de la paz o la sociedad global, por mencionar las más usuales. ${ }^{132}$ Como reflejo de esta variedad

\footnotetext{
${ }^{127}$ Ibid., pp. 273-274.

${ }^{128}$ El concepto apareció en el informe sobre Desarrollo Humano del PNUD de 1994 y, desde entonces, se utiliza para designar una adjetivación de la seguridad que se basa en la idea que la seguridad no solamente es un bien atribuible a los Estados, sino también a los individuos, quienes son asimismo sujetos de derecho internacional. De tal forma, la seguridad ya no es entendida sobre la base de la soberanía, territorialidad o poder militar, sino como un concepto multisectorial, interdependiente y, por último, como un derecho universal. Para ahondar en las similitudes y diferencias entre la seguridad comprehensiva y la seguridad humana vid. Acharya, Amitav (2002): "Human Security: What Kind for the Asia-Pacific?", en The Human Face of Security: Asia-Pacific Perspectives, Canberra, Strategic and Defence Studies Centre Australian National University, pp. 5-17.

${ }^{129}$ Ong, op. cit., p. 57.

${ }^{130}$ Do op. cit., pp. 173-174.

${ }^{131}$ Ibid. pp. 172-173.

${ }^{132}$ En las últimas décadas han aparecido numerosas iniciativas promovidas por instituciones tanto públicas como privadas sobre estos nuevos temas de investigación. A modo de ejemplo encontramos el Center for the Promotion of Disarmament and Non-Proliferation; el Council for Security Cooperation in the Asia Pacific y el Pacific Economic Cooperation Council. Estos proyectos han sido promovidos, entre otros, por el Japan Institute of International Affairs; el Hiroshima Peace Institute de la Hiroshima City University o la Global Peacebuilding Association.
} 
temática, es habitual encontrar en las universidades japonesas programas sobre estudios globales ${ }^{133}$ en los que se abordan diferentes dimensiones de la seguridad comprehensiva y humana, incluyendo cuestiones de seguridad nacional, desarrollo sostenible de comunidades y grupos culturales y/o étnicos, protección medioambiental, economía y negocios internacionales.

\subsection{La (ir)relevancia de las teorías japonesas en la política exterior de la administración de Shinzo Abe}

Algunos fundamentos meta-teóricos y prácticas que nutrieron la política exterior del Japón de preguerra han resonado en la diplomacia japonesa de posguerra. Esta continuidad puede encontrarse, al menos, en cuatro cuestiones. En primer lugar, en la revitalización del modelo de los gansos voladores tras el boom económico japonés de los años sesenta. Tras publicar su modelo de los gansos voladores en lengua inglesa en 1962, se dieron a conocer las explicaciones sobre el desarrollo económico en Asia, por lo que la popularidad de Akamatsu aumentó considerablemente entre los académicos occidentales.

En segundo lugar, observamos una línea de continuidad en la cuestión del fomento del regionalismo asiático. Desde 1970 Japón ha renovado su interés por un regionalismo dirigido hacia Asia mediante la promoción de un gran número de iniciativas como el establecimiento de las relaciones con ASEAN (1973), la creación de APEC (1989), el fomento de los llamados "valores asiáticos" o la formación de una "Comunidad de Asia Oriental", concepto propuesto por el Primer Ministro Junichiro Koizumi en 2002 y, posteriormente adoptado por el Primer Ministro Yukio Hatoyama en 2009. ${ }^{134}$ Ahora bien, si el regionalismo japonés de los años setenta y ochenta se fundamentaba en una cierta diferenciación respecto a Occidente, en la actualidad Japón ha promovido iniciativas de integración económica y política que superan el ámbito asiático. A modo de ejemplo, Japón ha promovido la participación de los Estados Unidos y Rusia en la Cumbre de Asia Oriental, ha formado parte en las negociaciones del Trans-Pacific Partnership —que incluye a otras naciones como México, Perú, Canadá o los mismos Estados Unidos - y ha fomentado acuerdos de libre comercio con las principales economías occidentales como la Unión Europea (2018) o con los Estados Unidos —en negociación—.

En tercer lugar, Shimiz ${ }^{135}$ argumenta que existe cierta continuidad en las justificaciones en materia de política exterior basadas en razonamientos culturalistas. Este autor destaca cómo la Escuela de Kyoto, durante el Japón imperial, identificó a Occidente como el origen de las crisis que vivía la humanidad de la época. En aquel entonces, los valores japoneses se presentaron como salvaguarda ante los peligros que acechaban a la humanidad, lo cual resultó en una profunda diferenciación entre Oriente y Occidente en la retórica de los autores de la Escuela de Kyoto. Para Shimizu, los responsables políticos japoneses han mantenido esta aproximación de superioridad cultural en la implementación del poder blando japonés. Uno de los ejemplos de dicho poder blando japonés se encuentra en la idea que la sociedad japonesa muestra una aproximación respetuosa e integradora de con el medio ambiente, idea que sitúa al

\footnotetext{
${ }^{133}$ Los estudios globales analizan las relaciones transnacionales desde una perspectiva interdisciplinaria y están estrechamente vinculados con el concepto de seguridad humana y con parte de las teorías críticas de las RRII. Dichos estudios se oponen a la perspectiva estatocéntrica de las RRII, la cual es habitual en la disciplina. Para entender en detalle el concepto de estudios globales vid. Nederveen Pieterse, Jan: "What is Global Studies?" Globalizations, vol. 10, no 4 (agosto 2013), pp. 499-514.

${ }^{134}$ Do añade también el "Arco de libertad y prosperidad" propuesto en 2006 como ejemplo de este regionalismo. No obstante, el "Arco de libertad y prosperidad" se extendía geográficamente hasta integrar numerosos países europeos. Vid. Do op. cit. p. 154.

135 Shimizu, Kosuke: "The ambivalent relationship of Japan's soft power diplomacy and Princess Mononoke: Tosaka Jun's philosophy of culture as moral reflection", Japanese Journal of Political Science, vol. 15, n $\mathrm{n}^{\circ}$ (diciembre 2014).
} 
país en una posición de superioridad en relación con la tendencia agresiva con la naturaleza por parte Occidente. ${ }^{136}$

En consecuencia, los responsables políticos han tomado los valores japoneses como algo fijo, sin considerar que las culturas están constantemente mutando y relacionándose entre ellas, por lo que utilizan sus valores para aumentar la capacidad de influencia mediante el uso del poder blando japonés. Este poder blando busca esencializar los productos culturales japoneses con la intención de promover determinados objetivos políticos. En concreto, Shimizu destaca que la actual esencialización, estandarización cultural y falta de una reflexión crítica sobre los productos culturales japoneses es similar a la abstracción que se produjo por parte de la Escuela de Kyoto durante el Japón imperial.

Finalmente, el Japón de posguerra construyó una identidad pacifista esencialmente diferenciada al resto de Estados. ${ }^{137}$ Esta identidad se estimaba como positiva por una parte de la sociedad y estaba representada mayoritariamente por partidos de izquierdas o progresistas. En cambio, parte del conservadurismo japonés consideraba que el pacifismo convertía a Japón en un país "no normal" e ilegítimamente diferente. ${ }^{138}$ Ello resultó en la construcción de una identidad japonesa ambigua que daría lugar a un doble proceso de socialización y securitización. Por una parte, Japón internalizaba los planteamientos teóricos del mainstream de las RRII desde la excepcionalidad pacifista de Japón. Por otra parte, aquellos que consideraban negativamente la excepcionalidad japonesa promovieron un proceso de securitización para terminar con dicha anormalidad. La política exterior y de seguridad del gobierno de Shinzo Abe ${ }^{139}$ desde 2012 ha supuesto un avance en la dirección hacia dicha normalización.

En este avance hacia la normalización de la política de seguridad de Japón, la administración Abe ha promovido la supresión de los límites jurídicos-sociales que fueron impuestos a Japón tras la derrota en la Segunda Guerra Mundial, así como otras medidas autoimpuestas durante las décadas posteriores. Algunos de estos límites se encuentran en la renuncia al derecho de beligerancia del artículo 9 de la Constitución japonesa, el establecimiento de un techo presupuestario para la defensa en el uno por ciento del PIB, la aprobación de severas restricciones para la exportación de armamento o el reconocimiento de los principios no nucleares de no poseer, producir o introducir armamento nuclear en Japón.

Asimismo, la administración Abe ha aprobado múltiples iniciativas que otorgan a Japón mayores funciones y capacidades para la seguridad internacional. Entre las iniciativas adoptadas por el gobierno de Abe destacan la creación del Consejo de Seguridad Nacional para agilizar la toma de decisiones en materia de seguridad, el aumento significativo del presupuesto de defensa, la retirada de los límites a las exportaciones de armamento o la propuesta para reformar el artículo nueve de la Constitución japonesa. El gobierno de Abe no solamente ha incrementado las capacidades militares de Japón y levantado algunas de las limitaciones en materia de seguridad, sino que también ha actualizado, facilitado y mejorado la coordinación

\footnotetext{
136 Ibid.

137 Hagström, Linus: "The 'abnormal' state: Identity, norm/exception and Japan", European Journal of International Relations, vol. 21, $\mathrm{n}^{\mathrm{o}} 1$ (marzo 2015), pp. 137-138.

${ }^{138}$ Ibid. p. 129.

${ }^{139}$ Abe proviene de una familia con una larga tradición política. Su abuelo materno fue el exprimer ministro Kishi (1957-1960), quién había ocupado cargos de alta responsabilidad en los gobiernos del Japón imperial desde finales de los años treinta, es decir, durante la formulación y auge de la Gran Esfera de Cooperación de Asia Oriental. Tras haber sido encarcelado durante la ocupación estadounidense, Kishi fue liberado y devino uno de los cuadros destacados del principal partido del Japón de posguerra, el Partido Liberal Democrático. Entre las iniciativas más destacadas de su mandato como Primer Ministro se encuentra la renegociación del Tratado de Seguridad con los Estados Unidos, en el que se incrementaba la cooperación para la seguridad entre ambos Estados y obligaba a Washington a asegurar la defensa del territorio japonés.
} 
en materia seguridad con los Estados Unidos. En esta dirección, se ha reinterpretado en 2015 la Constitución para permitir la legítima defensa colectiva en aquellas situaciones en que la seguridad de Japón pueda estar comprometida, lo que supone una referencia implícita a un eventual apoyo militar a los Estados Unidos.

En suma, a pesar de impulsar el proceso de normalización de Japón y mantener cierto esencialismo cultural en la promoción del poder blando japonés, la política exterior y de seguridad de la administración Abe difiere tanto del modelo de regionalismo asiático del Japón imperial como de la formulación de una identidad japonesa contrapuesta a Occidente. Ello se explica por qué la política exterior y de seguridad de Abe se ha fundamentado en el fortalecimiento de la cooperación para la defensa también con países de Occidente y en la promoción de un regionalismo con marcos de integración trans- o interregionales.

\section{Conclusiones}

En este artículo hemos constatado una aseveración cada vez más aceptada por los teóricos de la disciplina de las RRII: su carácter eminentemente occidentalcéntrico y la situación de inferioridad o, cuanto menos, de poca visibilidad de la teoría no occidental. Como apuntábamos en la introducción, esta situación ha sido explicada por numerosos teóricos como el resultado del predominio de Europa y Norteamérica en las Ciencias Sociales, una dominación producto del estatus hegemónico conseguido por las potencias occidentales. Aunque la teoría no occidental puede entenderse como el resultado de un proceso de dominación, en algunos casos de cariz colonial, las academias locales también han jugado un papel relevante a la hora de asimilar o combatir dicho dominio. Dicho en términos propios del debate agente-estructura, los condicionantes sistémicos o las circunstancias históricas no explican cualquier fenómeno de las RRII; por el contrario, es necesario escudriñar los agentes de dichas relaciones.

Para contribuir al debate, hemos analizado dos casos cuya relevancia trasciende la de su contexto regional: China y Japón. En el caso de academia china, hemos identificado la existencia de una Escuela china de RRII heterogénea. En efecto, la producción de teoría china está conformada por un conjunto de teorías, en algunos casos, contrapuestas, que se encuentran en un proceso de refinamiento. Además, en el seno de la academia china existen hondas discusiones sobre cómo se debe teorizar y cuál es su objetivo último.

Nuestro análisis revela que la reciente notoriedad del proyecto de la Escuela china de RRII se explica por tres factores claves: por la capacidad de sus teóricos para formular razonamientos teóricos complejos fundamentados en la filosofía de la ciencia occidental; por el reconocimiento internacional de las aportaciones a la teoría general de las RRII por parte de algunos de sus académicos destacados, y por el giro pospositivista que ha experimentado la disciplina. Esta preferencia por la teoría pospositivista se explica tanto por la reestructuración de un sistema internacional cada vez más multipolar como por el cuestionamiento de teorías que se consideraban universales, como el concepto del equilibrio de poder o el de anarquía.

En suma, la ontología y la epistemología de cada una de estas contribuciones teóricas se sitúan en diferentes puntos de partida. Mientras que la teoría Tianxia y el Realismo Moral son claramente positivistas, la "Teoría de la Relacionalidad" la ubicamos en un estadio intermedio entre el positivismo y el pospositivismo. Todo ello nos reafirma en lo que apuntábamos apriorísticamente en la introducción: la Escuela china, además de poner en cuestión los cimientos de la disciplina occidentalcéntrica, ha empezado a cuestionar sus ideas y normas e, incluso, a combatir su hegemonía.

En el caso de Japón, hemos analizado el surgimiento y la evolución de la disciplina de las RRII en dicho país, enfatizando la aparición de los grandes debates en la academia japonesa y hemos observado si dicha academia se ha desarrollado siguiendo unos parámetros diferentes 
de Occidente. Tras la victoria aliada en la Primera Guerra Mundial, Japón no obtuvo el reconocimiento y las concesiones esperadas, por lo que pretendió reafirmarse como gran potencia y establecer su propia manera de entender la sociedad internacional. En este contexto se teorizó sobre una Esfera de Coprosperidad de la Gran Asia Oriental que acabó instrumentalizándose por el poder político para alcanzar los objetivos y ambiciones del país. En el caso del modelo de "gansos voladores", a pesar de que su teorización la situamos en el periodo de entreguerras, recobra importancia en los años sesenta, cuando Japón compite como poder económico global con otras potencias.

Hemos caracterizado el período posterior a la Segunda Guerra Mundial como el que ha permitido una incorporación progresiva de la epistemología y ontología de la academia occidental en Japón. Ello, sin menoscabar la creación de conceptos originales como la seguridad comprehensiva, que acabará impregnando la evolución del concepto de seguridad a nivel global. Todo ello nos permite concluir que Japón avanza en el desarrollo teórico en los momentos en que el país se posiciona como potencia en ascenso. Visto desde la perspectiva de quienes proponen las normas globales, Japón produce teoría en los momentos en que cuestiona el statu quo existente. En cambio, en períodos de acomodo con el statu quo, como en la actualidad (período de Shinzo Abe), se manifiesta una falta de desarrollo teórico con particularidades autóctonas.

En conclusión, este estudio constata una idea cada vez más generalizada en la academia occidental: el cambio en la distribución del poder en un sistema internacional en plena transformación está afectando ya a la teorización de las RRII. En especial, el papel de China como potencia en ascenso y de Japón como gran potencia en declive requieren de un cambio de cariz teórica. Sin embargo, esta necesidad no solamente se explica como el resultado de un sistema de poder en mutación. La visualización de algunas teorías autóctonas está generando un conocimiento cuya incorporación en el mainstream resulta ineludible para la consecución de una disciplina más plural e inclusiva. Los diferentes marcos analíticos que emergen más allá de los confines de Norteamérica y Europa, además de desenmascarar su carácter hegemónico y de desnudar su provincialismo, también ofrecen interpretaciones rigurosas de la realidad internacional. No obstante, la producción de un conocimiento más plural para la disciplina e inclusivo para las diferentes cosmovisiones culturales no menoscaba la búsqueda de elementos universales en las diferentes teorías que conforman las RRII.

\section{Bibliografía}

Acharya, Amitav (2002): "Human Security: What Kind for the Asia-Pacific?", en The Human Face of Security: Asia-Pacific Perspectives, Canberra, Strategic and Defence Studies Centre Australian National University, pp. 5-17.

Acharya, Amitav: "Theorising the international relations of Asia: necessity or indulgence? Some Reflections". The Pacific Review, vol. 30, nº (noviembre 2017), pp. 816-828.

Acharya, Amitav y Buzan, Barry: "Why is there no non-Western international relations theory? An introduction", International Relations of the Asia-Pacific, vol. 7, no 3 (septiembre 2007), pp. 287-312.

Acharya, Amitav y Buzan, Barry (eds.) (2010): Non-Western International Relations Theory: perspectives on and beyond Asia, London, Routledge. 
Acharya, Amitav y Buzan, Barry: "Why is there no Non-Western International Relations Theory? Ten years on", International Relations of the Asia-Pacific, vol. 17, $\mathrm{n}^{\mathrm{o}} 3$ (septiembre 2017).

Agathangelou, Anna M. y Ling, L. H. M.: "The House of IR: From Family Power Politics to the Poisies of Worldism", International Studies Review, vol. 6, no 4 (diciembre 2004), pp. 2150 .

Akamatsu, Kaname: “A Historical Pattern of Economic Growth in Developing countries", The Developing Economies, vol. 1, (agosto 1962), pp. 3-25.

Arenal, Celestino. (2014): Etnocentrismo y Teoría de las Relaciones Internacionales: una visión crítica, Madrid, Editorial Tecnos.

Arenal, Celestino y Sanahuja, José Antonio (eds.) (2015): Teorías de las Relaciones Internacionales, Madrid, Tecnos.

Arisaka, Yoko: “The Nishida Enigma: 'The Principle of the New World Order", Monumenta Nipponica, vol. 51, no 1 (verano 1996), pp. 81-105.

Asada, Sadao (1988): Japan and the World, 1853-1952: A Bibliographic Guide to Recent Scholarship in Japanese Foreign Relations, New York, Columbia University Press.

Buckley, Chris: "Xi Jinping thought explained: a new ideology for a new era", New York Times, 26 de febrero de 2018, en https://www.nytimes.com/2018/02/26/world/asia/xi-jinpingthought-explained-a-new-ideology-for-a-new-era.html.

Callahan, William A.: "China and the Globalization of IR Theory: discussion of 'Building International Relations Theory with Chinese Characteristics"', Journal of Contemporary China, vol. 10, no 26 (febrero 2001), pp. 75-88.

Callahan, William A.: "Chinese Visions of World Order: Post-hegemonic or a New Hegemony?", International Studies Review, vol. 10, no 4 (diciembre 2008), pp. 749-761.

Chan, Gerald: "International Studies in China: origins and development",Issues and Studies, vol. 33, no 2 (febrero 1997), pp. 40-64.

Chan, Gerald (1999): Chinese Perspectives on International Relations: a Framework for Analysis, New York, St. Martin's Press, pp. 375-403.

Chan, Stephen y Mandaville, Peter G. (eds.) (2001): The Zen of International Relations. IR Theory from East to West, London, Palgrave Macmillan.

Chang, En-Chung; Chou Ting-Jui; Huang, Chungming y Wang, Xun: "The Categories, Rules, and Demonstrations of Guanxi in Chinese Society",Journal of Business-to-Business Marketing, vol. 23, no 4 (octubre 2016), pp. 311-325.

Chen, Ching-Chang: "The Im/Possibility of Building Indigenous Theories in a Hegemonic Discipline: The Case of Japanese International Relations", Asian Perspective, vol. 36, $\mathrm{n}^{\circ} 3$ (julio-septiembre 2012), pp. 463-492.

Colegrove, Kenneth: “The New Order in East Asia”, The Far Eastern Quarterly, vol. 1, n 1 (noviembre 1941), pp. 5-24.

Corrêa Malafaia, Thiago: "Japanese International Relations: An assessment of the 1971-2011 period", Brazilian Political Science Review, vol. 10, nº 1 (abril 2016), pp. 1-28.

Cox, Robert W. "Social Forces, States and World Orders: Beyond International Relations Theory", Millennium, vol. 10, nº 2 (junio 1981), pp. 126-155. 
Cunningham-Cross, Linsay: "Using the past to (re)write the future: Yan Xuetong, pre-Qin thought and the China's rise to power", China Information, vol. 26, n 2 (junio 2012), pp. 219233.

Cunningham-Cross, Linsay (2014): In search of a Chinese School: Ghostly encounters with the parochial/global discipline of international relations, University of Manchester [tesis doctoral].

Deng, Yong: “The Chinese Conception of National Interests in International Relations", The China Quarterly, no 154 (junio 1998), pp. 308-329.

Dirlik, Arif y Zhang, Xudong: "Introduction: Postmodernism and China”, Boundary 2, vol. 24, n 3 (otoño 1997), pp. 1-18.

Do, Thuy T.: "China's rise and the 'Chinese dream' in international relations theory", Global Change, Peace \& Security, vol. 27, nº 1 (Febrero 2015), pp. 21-38.

Do, Thuy T. (2016): The practices of knowledge claims: Reflections from the drive toward constructing "East Asian International Relations Theory". The Australian National University [tesis doctoral].

Doty, Roxanne Lynn (1996): Imperial Encounters: the Politics of Representation in NorthSouth Relations, Minneapolis, University of Minnesota Press.

Galindo Rodríguez, Fernando: "Enfoques postcoloniales en Relaciones Internacionales: un breve recorrido por sus debates y sus desarrollos teóricos", Relaciones Internacionales, vol. 22 (febrero 2013), pp. 85-107.

Geeraerts, Gustaaf y Jing Men: "International Relations Theory in China", Global Society, vol. $15, \mathrm{n}^{\circ} 3$ (mayo 2001), pp. 251-276.

Goh, Sui Noi:"19th Party Congress: Xi Jinping outlines new thought on socialism with Chinese traits", The Strait Times, 28 de octubre de 2017, en https://www.straitstimes.com/asia/eastasia/19th-party-congress-xi-jinping-outlines-new-thought-on-socialism-with-chinese-traits

Goto-Jones, Christopher S. (2005): Political Philosophy in Japan: Nishida, the Kyoto School and Co-prosperity, London, Routledge.

Gruffydd Jones, Branwen (ed.) (2006): Decolonizing International Relations, Lanham, Rowman \& Littlefield.

Hagström, Linus: "The 'abnormal' state: Identity, norm/exception and Japan", European Journal of International Relations, vol. 21, $\mathrm{n}^{\mathrm{o}} 1$ (marzo 2015), pp. 122-145.

Hobson, John M. (2012): The Eurocentric Conception of World Politics: Western International Theory, 1760-2010, New York, Cambridge University Press.

$\mathrm{Hu}$, Jintao: "Build towards a Harmonious World of Lasting Peace and Common Prosperity", en Discurso ante la cumbre de las Naciones Unidas en New York (septiembre 2005) http://www.un.org/webcast/summit2005/statements15/china050915eng.pdf

Huang, Chiung-Chiu y Shih, Chih-yu (2014): Harmonious intervention: China's quest for relational security, Farnham, Ashgate.

Ikeda, Josuke: "Japanese Vision of International Society: A Historical Exploration", en Shimizu, Kosuke; Ikeda, Josuke; Kamino, Tomoya y Sato, Shiro (2008): Is There a Japanese IR? Seeking an Academic Bridge through Japan's History of International Relations, Otsu, Afrasian Centre for Peace and Development Studies, Ryukoku University, pp. 29-45.

Inoguchi, Takashi (1989): “The Study of International Relations”, en The Study of International Relations: The State of the Art, New York, Palgrave Macmillan, pp. 250-264. 
Inoguchi, Takashi: “Are there any Theories of International Relations in Japan?" International Relations of the Asia-Pacific, vol. 7, n 3 (septiembre 2007), pp. 369-390.

Inoguchi, Takashi y Bacon Paul: "The study of International Relations in Japan: towards a more International Discipline", International Relations of the Asia-Pacific, vol. 1, $\mathrm{n}^{\circ} 1$ (enero 2001), pp. 1-20.

Jintao, Hu: "Report of Hu Jintao to the 18th CPC National Congress", China.org.cn, 16 de noviembre de 2012, en http://www.china.org.cn/china/18th_cpc_congress/201211/16/content_27137540_11.htm

Kamino, Tomoya: The Twenty Years' Crisis, 1919-1939: An Introduction of the Study of International Relations in Japan", en Shimizu, Kosuke; Ikeda, Josuke; Kamino, Tomoya y Sato, Shiro (2008): Is There a Japanese IR? Seeking an Academic Bridge through Japan's History of International Relations, Otsu, Afrasian Centre for Peace and Development Studies, Ryukoku University, pp. 5-28.

Kasahara, Shigehisa: "The Flying Geese Paradigm: A Critical Study of Its Application to East Asian Regional Development", UNCTAD Discussion, no 169 (abril 2004).

Kim, Hun Joon: "Will IR theory with Chinese Characteristics be a Powerful Alternative?", The Chinese Journal of International Politics, vol. 9, $\mathrm{n}^{\circ} 1$ (marzo 2016), pp. 59-79.

Krishna, Sankaran: "The Importance of Being Ironic: A Postcolonial View on Critical International Relations Theory", Alternatives: Global, Local, Political, vol. 18, $\mathrm{n}^{\circ} 3$ (julio 1993), pp. 385-417.

Kobayashi, Hiroharu (2003): “Royama Masamichi's Perception of International Order from the 1920 s to 1930s and the Concept of the East Asian Community", en Nationalism and Internationalism in Imperial Japan: Autonomy, Asian Brotherhood, or World Citizenship?, New York, Routledge, pp. 135-168.

Kojima, Kiyoshi: "The "Flying Geese" Model of Asian Economic Development: Origin, Theoretical Extensions, and Regional Policy Implications", Journal of Asian Economics, vol. 11, nº 4 (otoño 2000), pp. 375-401.

Korhonen, Pekka: "The Theory of the Flying Geese Pattern of Development and Its Interpretations", Journal of Peace Research, vol. 31, no 1 (febrero 1994), pp. 93-108.

Kristensen, Peter Marcus y Zhang Yongjin: “The curious case of 'Schools' of IR: From the sociology of the geopolitics of knowledge", The Chinese Journal of International Politics, vol. 10, nº 4 (Invierno 2017), pp. 429-454.

Kuhn, Thomas S. (1962): The Structure of Scientific Revolutions, Chicago, University of Chicago Press.

Lakatos, Imre (1978): The Methodology of Scientific Research Programmes: Philosophical Papers Volume 1, Cambridge, Cambridge University Press.

Laudan, Larry (1978): Progress and its Problems: Towards a Theory of Scientific Growth, Los Angeles, University of California Press.

Li, Mao: "China Should Speak its Own Language, Scholars Say at IR Meeting”, Chinese Social Science Today, 25 de julio de 2013, en http://www.csstoday.com/Item/254.aspx

Li, Nan y Xie, Peng: "Guoji guanxi "zhongguo xuepai” de jiangou ji qi xiandai xing fansi [The construction of the "Chinese School" in International Relations and its reflection on modernity]", Journal of Shanxi Datong University (Social Science), vol. 33, nº 1 (febrero 2019), pp. 15-19. 
Liang, Shoude: "Lun Guoji zhengzhixue de zhongguo tese [On Chinese characteristics of International Politics studies]", Guoji Zhengzhi Yanjiu, no 1 (enero-marzo 1994), pp. 15-21.

Liang, Shoude: "Constructing an International Relations Theory with "Chinese Characteristics"”, Political Science, vol. 49, nº 1 (julio 1997), pp. 23-39.

Ling, L. H. M. (2002): Postcolonial International Relations: Conquest and Desire between Asia and the West, New York, Palgrave.

Lorenzini, María Elena y Pereyra Doval, María Gisela: "Revisitando los aportes de las teorías del sur: nexos entre teoría y praxis en Argentina y Brasil", Relaciones Internacionales, vol. 22 (febrero 2013), pp. 9-26.

Lu, Peng: "Pre-1949 Chinese IR: an Occluded History", Australian Journal of International Affairs, vol. 68, nº 2 (marzo 2014), pp. 133-155.

Lu, Peng: "Chinese IR Sino-centrism tradition and its influence on the Chinese School Movement", The Pacific Review, (April 2018), pp. 1-18.

Mardell, Jacob: The “'Community of Common Destiny' in Xi Jinping's New Era", The Diplomat, 25 de octubre de 2017, en https://thediplomat.com/2017/10/the-community-ofcommon-destiny-in-xi-jinpings-new-era/

Merton, Robert K. (1968): Social Theory and Social Structure, New York, Free Press.

Michael, Michaelis S. y Petito, Fabio (eds.) (2009): Civilizational Dialogue and World Order: The Other Politics of Cultures, Religions, and Civilizational in International Relations, Nueva York, Palgrave Macmillan.

Montobbio, Manuel: "Tianxia, Teoría de las Relaciones Internacionales y ascenso global de China", Revista Española de Derecho Internacional, vol. 70, nº 1 (enero-junio 2018), pp. 235246.

Moure, Leire (2014): "Orden internacional en transición y Relaciones Internacionales: Aproximaciones teóricas al declive hegemónico estadounidense y al ascenso de China como potencia global", en Cursos de Derecho Internacional y Relaciones Internacionales de VitoriaGasteiz 2013, Madrid, Thompson Reuters-Aranzadi.

Nakano, Ryoko: “'Pre-History’ of International Relations in Japan: Yanaihara Tadao's Dual Perspective of Empire”, Millennium, vol. 35, nº 2 (marzo 2007), pp. 301-319.

Nakano, Ryoko (2018): "Rethinking the Liberal/Pluralist Vision of Japan's Colonial Studies", en Modern Japanese Political Thought and International Relations, London, Rowman \& Littlefield International.

Nederveen Pieterse, Jan: "What is Global Studies?” Globalizations, vol. 10, n 4 (agosto 2013), pp. 499-514.

$\mathrm{Ni}$, Chang-wen: "guanyu guoji guanxi xue "san da fanshi" de fansi - yi bijiao zhengzhi xue he lishi shehui xue jiangou "zhongguo xuepai" [Reflections on the "Three theories" of International Relations - constructing the "Chinese School" in the light of comparative politics and historical sociology]", Jiangnan Shehui Xueyuan Xuebao, vol. 19, n 2 (junio 2017), pp. 49-54.

Noesselt, Nele: "Revisiting the Debate on Constructing a Theory of International Relations with Chinese Characteristics", The China Quarterly, n 222 (junio 2015), pp. 430-448.

Ong, Graham Gerard: “Building an IR Theory with 'Japanese Characteristics': Nishida Kitaro and 'Emptiness"”, Millennium, vol. 33, n 1 (enero 2004), pp. 35-58. 
Ozawa, Terutomo: "The "Hidden" Side of the "Flying-Geese" Catch-up Model: Japan's Dirigiste Institutional Setup and a Deepening Financial Morass", Journal of Asian Economics, vol. 12, no 4 (invierno 2001), pp. 471-491.

Peters, Michael A.: "The Chinese Dream: Xi Jinping thought on socialism with Chinese characteristics for a new era", Educational Philosophy and Theory, vol. 49, nº 14 (noviembre 2017), pp. 1299-1304.

Pintado Lobato, Montse: "Hacia una teoría china de las relaciones internacionales: Evolución, proyectos teóricos y pertinencia práctica", Revista Española de de Derecho Internacional, vol. 70, no 1 (enero-junio 2018), pp. 201-226.

Qin, Yaqing: "Why is there no Chinese International Relations Theory?" International Relations of the Asia-Pacific, vol. 7, no 3 (septiembre 2007), pp. 313-340.

Qin, Yaqing: "Development of International Relations Theory in China”, International Studies, vol. 46, n 1-2 (enero 2009), pp. 185-201.

Qin, Yaqing: "Relationality and processual construction: bringing Chinese ideas into international relations theory", Social Sciences in China, vol. 30, no 3 (agosto 2009), pp. 5-20.

Qin, Yaqing: "Development of International Relations theory in China: Progress through Debates", International Relations of the Asia-Pacific, vol. 11, no 2 (mayo 2011), pp. 231-257.

Qin, Yaqing: "Cultura y pensamiento global: una Teoría china de las Relaciones Internacionales", Documentos CIDOB Asia, no 28 (septiembre 2013), pp. 67-90.

Qin, Yaqing (2018): A relational theory of World politics, Cambridge, Cambridge University Press.

Qiong, Liu: "Xijinping: Rang mingyun gongtongti yishi zhai zhoubian guojia luodi Shenggen [Xi Jinping: Let the community of common destiny take root in neighboring countries], Xinhua Net, 25 de octubre de 2013, en http://www.xinhuanet.com//politics/201310/25/c_117878944.htm

Ran, Mei: "Gai bu gai you guoji zhengzhi lilun de zhongguo xuepai - jian ping meiguo de guoji zhengzhi lilun" [Should There be a Chinese School of IR Theory? Remarks on American Theories of International Politics]", Guoji Zhengzhi Yanjiu, no 1 (enero-marzo 2000), pp. 6367.

Ren, Xiao: “'Lilun yu guoji guanxi lilun: yixie sikao' [Theory and IR Theory: Some Thoughts]", Chinese Journal of European Studies, nº 4 (julio-agosto 2000), pp. 19-25.

Said, Edward W. (1979): Orientalism, Nueva York, First Vintage Books Edition.

Secure a Decisive Victory in Building a Moderately Prosperous Society in All Respects and Strive for the Great Success of Socialism with Chinese Characteristics for a New Era, en http://www.xinhuanet.com/english/download/Xi_Jinping's_report_at_19th_CPC_National_Co ngress.pdf

Seth, Sanjay (ed.) (2013): Postcolonial Theory and International Relations: a Critical Introduction, London, Routledge.

Sheng, Ding: "To build a "harmonious world": China's soft power wielding in the global south", Journal of Chinese Political Science, vol. 13, nº 2 (agosto 2008), pp. 193-213.

Shimizu, Kosuke: “Culture and International Relations: Why Do Japan's IR Focus on the Third Dimension?", en Shimizu, Kosuke; Ikeda, Josuke; Kamino, Tomoya y Sato, Shiro (2008): Is There a Japanese IR? Seeking an Academic Bridge through Japan's History of International 
Relations, Otsu, Afrasian Centre for Peace and Development Studies, Ryukoku University, pp. 69-83.

Shimizu, Kosuke: "Nishida Kitaro and Japan's interwar foreign policy: War involvement and culturalist political discourse", International Relations of the Asia-Pacific, vol. 11, $\mathrm{n}^{\mathrm{o}} 1$ (enero, 2011), pp. 157-183.

Shimizu, Kosuke: "Materializing the "non-Western": two stories of Japanese Philosophers on Culture and Politics in the Inter-War Period", Cambridge Review of International Affairs, vol. 28, no 1 (julio 2014), pp. 3-20.

Shimizu, Kosuke: "The ambivalent relationship of Japan's soft power diplomacy and Princess Mononoke: Tosaka Jun's philosophy of culture as moral reflection", Japanese Journal of Political Science, vol. 15, no 4 (diciembre 2014), pp. 683-698.

Shimizu, Kosuke: "Materializing the 'non-Western': two stories of Japanese philosophers on culture and politics in the inter-war period", Cambridge Review of International Affairs, vol. $28, \mathrm{n}^{\mathrm{o}} 1(2015)$.

Shimizu, Kosuke: "The genealogy of culturalist international relations in Japan and its implications for post-Western discourse", All Azimuth, vol. 7, nº 1 (enero 2018), pp. 121-136.

Smith, Stephen N.: "Community of common destiny: China's "new assertiveness" and the changing Asian order", International Journal, vol. 73, n 3 (septiembre 2018), pp. 449-463.

Song, Xinning: "International Relations Theory-Building in China”, Political Science, vol. 49, no 1 (julio 1997), pp. 40-61.

Song, Xinning: "Building International Relations Theory with Chinese Characteristics", Journal of Contemporary China, vol. 10, $\mathrm{n}^{\circ} 26$ (febrero 2001), pp. 61-74.

Sun, Jisheng: "gaige kaifang yilai zhongguo guoji guanxi lilun fazhan huayu shijian yu chuangxin [Chinese IR theory development since China's reform and opening up: discourse, practice and innovation]", Shijie jingji yu zhengzhi, no 8 (junio 2018), pp. 4-29.

Tickner, Arlene B. y Wæver, Ole (eds.) (2009): International Relations Scholarship around the World, New York, Routledge.

Tickner, Arlene B. y Blaney, David. L. (eds.) (2012): Thinking International Relations Differently, New York, Routledge.

Tickner, Arlene B.: "Core, periphery and (neo)imperialist International Relations", European Journal of International Relations, vol. 19, nº 3 (septiembre 2013), pp. 627-646.

Tosa, Hiroyuki (2018): "The Pitfalls in the Project of Overcoming Western Modernity: Rethinking the Lineage of Japanese Historical Revisionism", en Modern Japanese Political Thought and International Relations, London, Rowman \& Littlefield International, pp. 111128.

Wang, Jianwei: "International Relations Studies in China”, Journal of East Asian Studies, vol. 2, nº 1 (febrero 2002), pp. 69-87.

Wang, Yiwei: "Between Science and Art: Questionable International Relations Theories", Japanese Journal of Political Science, vol. 8, $\mathrm{n}^{\circ} 2$ (agosto 2007), pp. 191-208.

Wight, Martin, Wight, Gabriele (ed.) y Porter, Brian (ed.) (1992): International theory: the Three Traditions, New York, Holmes \& Meier. 
Yamamoto, Kazuya: "International Relations Studies and Theories in Japan: a Trajectory Shaped by War, Pacifism, and Globalization", International Relations of the Asia-Pacific, vol. 11, no 2 (mayo 2011), pp. 259-278.

Wemheuer-Vogelaar, et al. "The IR of the beholder: examining global IR using the 2014 TRIP Survey", International Studies Review, vol. 18, nº 1 (marzo 2016), pp. 16-32.

Yan, Dexue: "Xijiping waijiao sixiang yu zhongguo tese guoji guanxi lilun goujian [Xi Jinping's thought on diplomacy and the construction of IR theories]", Dongbei Ya Luntan, ${ }^{\circ} 1$ (febrero 2019), pp.3-20.

Yan, Xuetong; Bell, Daniel A. (ed.); Sun, Zhe (ed.) y Ryden, Edmund (trad.) (2011): Ancient Chinese Thought, Modern Chinese Power, Princeton, Princeton University Press.

Yan, Xuetong: "Political Leadership and Power Redistribution", The Chinese Journal of International Politics, vol. 9, $\mathrm{n}^{\mathrm{o}} 1$ (marzo 2016), pp. 1-26.

Zhang, Chunman: "How to merge Western theories and Chinese indigenous theories to study Chinese politics", Journal of Chinese Political Science, vol. 22, n² (junio 2017), pp. 283-294.

Zhang, Denghua: “The concept of 'Community of Common Destiny' in China's Diplomacy: Meaning, Motives and Implications", Asia \& the Pacific Policy Studies, vol. 5, n 2 (enero 2018), pp. 196-207.

Zhang, Feng (2012): "Debating the "Chinese Theory of International Relations": Toward a New Stage in China's International studies", en Dallmayr, Fred y Zhao, Tingyang (eds.): In Contemporary Chinese Political Thought. Debates and Perspectives. Lexington, University of Kentucky Press, pp. 67-87.

Zhang, Feng: "The Tsinghua Approach and the Inception of Chinese Theories of International Relations", The Chinese Journal of International Politics, vol. 5, no 1 (marzo 2012), pp. 73102.

Zhao, Tingyang: "Rethinking Empire from a Chinese Concept "All-under-Heaven" (Tian-xia)", Social Identities, vol. 12, no 1 (enero 2006), pp. 29-41.

Zhao, Tingyang: “A Political World Philosophy in terms of All-under-heaven (Tianxia)", Diogenes, $\mathrm{n}^{\mathrm{o}} 221$ (febrero 2009), pp. 5-18.

Zhao, Tingyang (2018): Tianxia tout sous un même ciel. L'ordre du monde dans le passé et pour le futur, Paris, Les Éditions du Cerf.

Zhang, Yongjin, y Chang, Teng-Chi (eds.) (2016): Constructing a Chinese School of International Relations: Ongoing Debates and Sociological Realities, London, Routledge. 